\title{
Sound scattering by rough elongated elastic objects. I: Means of scattered field
}

\author{
T.K. Stanton \\ Department of Applied Ocean Physics and Engineering, Woods Hole Oceanographic Institution, \\ Woods Hole, Massachusetts 02543
}

(Received 28 August 1990; revised 9 October 1991; accepted 24 January 1992)

By use of the recently published deformed cylinder formulation [T. K. Stanton, J. Acoust. Soc. Am. 86, 691-705 (1989)], the scattered field due to rough elongated dense elastic objects is derived. The (one-dimensional) roughness is characterized by axial variations of radius. Explicit expressions are derived describing both the mean and mean square of the stochastic scattered field for the rough straight finite length cylinder (broadside incidence) for both $k a \ll 1$ and $k a \gg 1$ ( $k$ is the acoustic wave number and $a$ is the radius) while only the mean is calculated for the prolate spheroid, uniformly bent finite cylinder, and infinitely long cylinder for $k a \gg 1$ (again, all broadside incidence). The modal-series-based solution is used in the $k a \ll l$ case as the modal solution simplifies to the sum of two terms (monopole and dipole-like terms). For $k a \gg 1$, a more convenient approximate "ray" solution is used in place of the modal series solution. The results show that (1) when $k a \ll 1$ the roughness-induced variations of the mean and mean-square scattered fields due to the rough straight finite cylinder depend on the roughness, but are independent of frequency - an effect that has no counterpart in the area of scattering by rough planar interfaces. (2) When $k a \gg 1$ the mean specular (geometrically reflected) and Rayleigh surface elastic waves of the scattered field of each object are attenuated due to the roughness and their variations are dependent upon the frequency. In addition, the (roughness-induced) attenuation of the Rayleigh wave depends on the number of times the wave has circumnavigated the object. The mean-square values for the straight finite cylinder are attenuated in a similar manner with the additional dependence upon the correlation distance of the surface.

PACS numbers: 43.30.Ft, 43.30.Gv, 43.30.Hw

\section{LIST OF SYMBOLS}

$k \quad$ acoustic wave number $(=2 \pi / \lambda)$

a mean radius of cylinder cross section

$a_{\mathrm{RC}} \quad$ radius of cross section of rough cylinder

$\sigma \quad$ rms roughness relative to mean surface $\left(\equiv\left\langle\zeta^{2}\right\rangle^{1 / 2}\right)$

$P_{0} \quad$ amplitude of incident plane wave

$b_{m} \quad$ coefficient determined from boundary conditions

$H_{m}^{(1)} \quad$ Bessel function of the third kind (Hankel function of the first kind) of order $m$

$\lambda \quad$ wavelength of sound in surrounding fluid

$r \quad$ distance from axis of cylinder to the field point (applied to infinite and finite bodies).

$\phi \quad$ azimuthal angle ( $\phi=\pi$ is the backscatter direction)

$i \quad \sqrt{-1}$

$f^{(\infty)} \quad$ form function for infinitely long cylinder (subscripts $s, t w, R$, and $\lambda$, to $f^{(\infty)}$ stand for specular, transmitted wave, Rayleigh wave, and Whispering Gallery and Franz waves, respectively.) 


\section{$k \sin \theta$}

\section{$\hat{r}_{i}, \hat{r}_{r}$}

$L \quad$ length of straight finite object; arc length of uniformly bent cylinder

$\zeta \quad$ roughness perturbation about mean radius $a$

$\langle\cdots\rangle$ statistical ensemble average at fixed $\mathbf{r}_{\text {pos }}$ position distance along axis of straight cylinder and prolate spheroid ( $z$ axis is lengthwise axis of each straight object in this case)

$f_{0}$ scattering amplitude for perfectly smooth finite cylinder bivariate Gaussian PDF

normalized autocorrelation function of rough surface (it varies along lengthwise axis of body) correlation parameter of rough surface $\left(\equiv z^{\prime \prime}-z^{\prime}\right)$

correlation distance of rough surface probability density function

$\zeta\left(z^{\prime}\right), \xi\left(z^{\prime \prime}\right)$ length of semi-minor axis of prolate spheroid radius of curvature of axis of uniformly bent cylinder Sommerfeld-Watson transformation single variable Gaussian PDF

\section{INTRODUCTION}

There is a variety of volumetric objects in the ocean that scatter sound such as bubbles and marine organisms. Other objects that are associated with planar boundaries, such as ice keels and sand ripples, can be analyzed in volumetric terms in "boss" formulations such as that described by Twersky. ${ }^{1,2}$ Regardless of the type of scatterer the size, shape, orientation, and morphology must be thoroughly understood before predictions can be made. Research has typically involved use of very simple models to describe scattering by complex objects such as marine organisms and grains of sand..$^{3-15}$ The exact solutions to the sphere, infinitely long cylinder, and spheroid have long been candidates in those problems and recently approximate solutions to the straight and uniformly bent finite length cylinder have demonstrated improvements in the scattering models for zooplankton and fish. $^{9-15}$

Regardless of the class of shape used in the above scattering problems, the object was always considered to resemble a simple mathematical shape with a perfectly smooth surface. In some recent articles by this author ${ }^{9,10}$ and Pieper and Holliday, ${ }^{4}$ it was shown that in order to model the scattering of sound by zooplankton, the exact scattering solution involving the simple shape had to be modified for there to be an optimum fit between the "theory" and data. This was true for models involving spheres, straight finite cylinders, and uniformly bent finite cylinders. The modification involved truncation of the modal series solution and it was hypothesized that because the objects were irregular and did not exactly resemble the ideal simple mathematical shapes, that the objects would not support the higher modes that were inherent in the simple theory. There are relatively few publications involving the scattering by irregular objects and the solutions to those are limited to analytical solutions involving deformations of spheres ${ }^{16,17}$ and "simple" cylinders (impenetrable and/or infinitely long), ${ }^{18-21}$ and numerical methods involving more complex bodies. ${ }^{22,23}$

Recently, an approximate analytical solution was derived that predicts the scattering by deformed cylinders of finite length. ${ }^{10}$ The theory was developed to describe the scattering by elongated objects whose properties such as the

cross-sectional radius and material composition may vary with respect to the lengthwise axis. In addition, the axis of the cylinder may be deformed. The approximation lies in the assumption that the boundary conditions at each position along the axis are the same as those of the infinitely long cylinder, i.e., each infinitesimal section of the cylinder behaves as if the neighboring sections are identical where, in fact, their properties are slowly varying. The solution is then the integral of these infinitesimal sections over a finite length. As a result, the formulation is only valid for angles of incidence and reception normal or nearly normal to all tangents of the axis and for objects with very high aspect ratios ( $\gtrsim: 1$ ). In that article there was excellent agreement between the deformed cylinder calculations and the exact spheroidal wave function solution when the scattering by prolate spheroids (aspect ratio of 5:1) was calculated. In addition to the calculations involving prolate spheroids, predictions were made for uniformly bent finite cylinders that agreed with predictions based on Helmholtz-Kirchhoff results under limiting conditions.

While there was exact comparison between limiting forms of the solutions and other theories, the comparison between the uniformly bent cylinder solution and laboratory data (taken from Ref. 3) involving backscattering from euphausiids (a type of zooplankton) required modifications in the approximate theory. The modal series solution was truncated before convergence for an optimum fit between the theory and data with the hypothesis being that each object did not perfectly resemble a smooth uniformly bent cylinder but was actually a slightly irregularly shaped object whose roughness was perhaps a perturbation of the smooth bent cylinder. For the optimum fit, the first six terms of the series were required while the remainder of the terms were not included in the summation. Although this approach is an approximation to the actual problem, it also represents an improvement over previous attempts to model the scattering of sound by zooplankton. For example, Pieper and Holliday $^{4}$ and Stanton ${ }^{9}$ truncated other solutions to include only the first two terms when modeling the plankton as spheres and straight finite length cylinders, respectively. Hence, as the shape of the mathematical model object approached the shape of the actual object, the scattering was more accurate- 


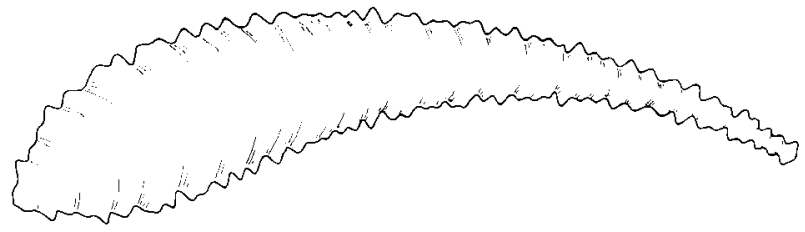

FIG. 1. Randomly rough elongated object (exaggerated roughness).

ly described with the accuracy being indicated by the number of terms retained in the series.

In this article, we formulate the deformed cylinder solution in such a way that it can analytically illustrate the effects of roughness on the scattering by rough elongated objects (Fig. 1). We chose dense elastic objects in this article because simple analytical solutions involving those boundary conditions are currently available to adapt to the case of rough objects. The essence of the approach lies in allowing the radius of the (circular) deformed cylinder to vary randomly along the length resulting in a one dimensional roughness. For $k a \ll 1$ the approximate solution is based on the modal series solution due to an infinitely long cylinder which simplifies to the sum of two terms-monopole and dipolelike terms-making the solution straight forward. For $k a \gg 1$, the modal series solution requires many terms to converge and is not mathematically convenient to manipulate. Therefore we apply a simplified "ray" solution in that region involving the Sommerfeld-Watson transformation (SWT) of the modal series solution which is much more convenient and physically relevant to use.

In Ref. 10, the modal series solution was used exclusively in the deformed cylinder solution and details of its use are given in that article. The SWT is applied to the deformed cylinder solution for the first time in this present article. The properties of the SWT and its use in the deformed cylinder formulation are summarized as follows: The SWT-based solution is a summation of rays due to the "specular" or geometrically reflected echo from the front surface of the object, Rayleigh and Whispering Gallery surface elastic waves, and Franz "creeping" surface waves as well as (internally) refracted waves. In our formulation, the "ray" solution is employed in the deformed cylinder solution so that the ray due to each infinitesimal element of the deformed cylinder is calculated with the phase of the rays being related to the roughness perturbations of the surface. All infinitesimal rays are then summed in the integral along the length of the object. The end result will be a backscattering cross section whose value depends upon the particular realization of rough surface. This value will vary from realization to realization due to the randomness of the phases of the rays.

Much work has appeared in the literature involving ray solutions ${ }^{24-45}$ ranging from the heuristically written simple solution by Veksler and Korsunskii ${ }^{44}$ to the more sophisticated SWT-based and Green's function solutions by a variety of investigators. Most important to this present analysis is a simple approximate formula for the "coupling coefficient" that predicts the amplitudes of the surface waves. We chose Marston's recently derived approximate formulas for use in our analysis as they demonstrate reasonable accuracy for the material (tungsten carbide) and range of $k a(15-40)$ chosen ${ }^{37-40}$ For simplicity we only analyze the case in which the specular and Rayleigh surface waves dominate the scattering. It also turns out that some of the scattering that results in narrow resonances which are ignored in the analysis becomes "washed away" due to roughness effects. One could use the procedure described in this article for analysis involving the other waves.

The general solution is used to derive explicit backscattering expressions for the following randomly rough objects at broadside incidence: straight finite length cylinder, prolate spheroid, uniformly bent finite cylinder, and infinitely long cylinder. Since the deformed cylinder solution is used as a basis, the solution is valid only for high aspect ratio objects ( $\gtrsim 5: 1)$ at angles of incidence at or near broadside. The roughness is modeled as continuous, stochastic, and, for simplicity, one dimensional (i.e., it depends only on the position with respect to the lengthwise axis). In order that the explicit expressions be evaluated analytically, the roughness was assumed to be Gaussian distributed with a linear autocorrelation function. The mean and mean-square scattered field is derived for both $k a \ll 1$ and $k a \gg 1$ for the straight finite cylinder while for the other shapes, only the mean field in the $k a \gg 1$ region is derived. For the sake of brevity and because of the mathematical difficulties involved in describing the mean-square field due to the other shapes for $k a \gg 1$, the other cases were not investigated. Because the vast majority of rough surface scattering articles in the literature involves rough planar interfaces, some general comparisons between the results in this article will be made with those articles. ${ }^{46-54}$ The case of axisymmetric roughness presented in this article results in effects separate and distinct from the case where the radius of the cylinder varies azimuthally. ${ }^{19-21}$ This article describes effects induced by roughness-related pathlength differences while the latter case involves variations in the coupling of the surface waves with the fluid.

This article, involving the mean and mean-square scattered field, is followed by an article describing the statistical behavior of the realization-to-realization fluctuations of the scattered signal. ${ }^{55}$ Application of the rough cylinder scattering theory to zooplankton scatter data is given in Ref. 14 where the statistics of the echoes from the animals is quantified in terms of the backscattering cross section of the animals.

\section{GENERAL SOLUTION}

\section{A. Infinitely long undeformed cylinder-Modal series and Sommerfeld-Watson transformation solutions}

The scattered pressure due to an infinitely long undeformed cylinder can be expressed as an infinite series of modal terms: ${ }^{56}$

$$
p_{\mathrm{scat}}=P_{0} \sum_{m=0}^{\infty} b_{m} i^{m} H_{m}^{(1)}(k r) \cos m \phi,
$$

where the $e^{-i w t}$ time dependence has been suppressed. This solution is exact for all $k r$ and $k a$.

In the far-field limit, the scattered pressure becomes

$$
p_{\text {scar }} \rightarrow P_{\mathrm{o}} \sqrt{a / 2 r} e^{i k r} f^{(\infty)}, \quad k r \gg 1 \text {, }
$$


where the form function $f^{(\infty)}$ is given in the modal series representation,

$$
\begin{aligned}
f_{M}^{(\infty)} & \equiv \frac{2}{\sqrt{\pi k a}} e^{-i \pi / 4} \sum_{m=0}^{\infty} b_{m} \cos m \phi \\
& =\frac{2}{\sqrt{\pi k a}} e^{-i \pi / 4} \sum_{m=0}^{\infty} b_{m}(-1)^{m} \quad \text { (backscatter) }
\end{aligned}
$$

The form function $f^{(\infty)}$ of this infinitely long cylinder should not be confused with the scattering amplitude $f$ used later in this article to describe the (spherically spreading) scattering by finite-sized objects. Both of these notations are consistent with the literature except for the fact that the " $\infty$ " sign refers in this case to the fact that the cylinder is infinitely long while elsewhere in the literature it may refer to the condition $k r \gg 1$. All results in this article involve the condition $k r \gg 1$ so the distinction is not made.

The above equations are the well-known modal series or partial wave series solution. While this solution has seen much use by a number of investigators, it requires many terms to converge mathematically in the $k a \gg 1$ region and is difficult to manipulate analytically. A convenient approximate form of the solution has been explored by use of the SWT, which allows a more physical interpretation of the scattered field at high frequencies by decomposing it into a sum of various rays such as the initial specularly "reflected" ray due to the front surface of the object $\left(f_{s}^{(\infty)}\right)$, internally refracted or transmitted (bulk) rays $\left(f_{i w}^{(\infty)}\right)$, Rayleigh $\left(f_{R}^{(\infty)}\right)$ and Whispering Gallery $\left(f_{\text {wo }}^{(\infty)}\right)$ surface elastic waves, and Franz creeping wave $\left(f_{F}^{(\infty)}\right):^{24-45}$

$$
f_{\mathrm{SWT}}^{(\infty)} \simeq f_{s}^{(\infty)}+f_{t w}^{(\infty)}+f_{R}^{(\infty)}+\sum_{l=W G, F} f_{\lambda_{l}}^{(\infty)} .
$$

Because of the complexity involved in taking into account all waves in the rough cylinder formulation, we limit this analysis to the materials and range of $k a$ where the specular and Rayleigh surface elastic waves dominate the scattering:

$$
f_{\mathrm{SWT}}^{(\infty)} \simeq f_{s}^{(\infty)}+f_{R}^{(\infty)}
$$

(conditionally valid, depending on material properties and $k a)$.

Including the other waves would require a case-by-case analysis using a procedure broadly similar to the one used in this article. The specular term is given simply in its $k a \gg 1$ limit:

$$
f_{s}^{(\infty)} \simeq \mathscr{R} e^{-i 2 k a},
$$

where the Rayleigh (plane-wave/plane-interface) reflection coefficient is given as

$$
\mathscr{R}=(z-1) /(z+1),
$$

where this " $\mathscr{R}$ " is not to be confused with subscript " $R$ " (Rayleigh surface wave) in other terms.

Expressions for $f_{R}^{(\infty)}$ are much more complex and are of the form:

$$
\begin{aligned}
f_{R}^{(\infty)}= & -G_{R} e^{-2\left(\pi-\theta_{R}\right) \beta_{R}} e^{i \eta_{R}} \\
& \times \sum_{m=0}^{\infty} e^{-2 \pi m \beta_{R}} e^{i 2 \pi m k a c / c_{R}}
\end{aligned}
$$

(note that the scattering by other surface waves can also be described by this form of equation ). The "launching" angle $\theta_{R}$ and phase shift term $\eta_{R}$ are given as

$$
\begin{aligned}
& \theta_{R}=\sin ^{-1}\left(c / c_{R}\right), \\
& \eta_{R}=k a\left[\left(c / c_{R}\right)\left(2 \pi-2 \theta_{R}\right)-2 \cos \theta_{R}\right],
\end{aligned}
$$

where $\theta_{R}$ is the local angle of incidence of the curved surface at which point the phase velocity of the Rayleigh surface wave matches the component of the phase velocity of the incident acoustic wave along the cylinder's surface.

In order to calculate the form function, $G_{R}$ and $\beta_{R}$ must be known. From Marston, we use the following approximate relationship: ${ }^{40}$

$$
\left|G_{R}\right| \simeq 8 \pi \beta_{R} / \sqrt{\pi k a}
$$

Since the phase of $G_{R}$ has been found to be approximately constant ${ }^{57}$ for cylinders, we can write $G_{R}$ as

$$
G_{R} \simeq 8 \pi \beta_{R} e^{i \Phi_{R}} / \sqrt{\pi k a} .
$$

Note that in Ref. 57, $\Phi_{R}$ was found to be approximately equal to $\pi / 4$ for $k a \gg 1$, although the general form of $G_{R}$ will be retained until evaluation in Sec. IV. Also, the dispersive characteristics of $c_{R}$ are ignored in the above expressions. As illustrated in Ref. 39, the dependence of $c_{R}$ upon $k a$ is especially important for values of $k a$ below 15 , hence limiting the analysis to $k a \gtrsim 15$. Marston also showed that $\beta_{R}$ can be expressed in a simple approximate manner:

$$
\beta_{R} \simeq k a \beta_{R}^{\prime}(\infty), \quad k a \gg 1 \text {. }
$$

For tungsten carbide, $\beta_{R}^{\prime}(\infty)$ is equal to 0.00232 . The above relationship produces reasonable results in the $15<k a<40$ range for tungsten carbide. Thus, at least for a range of $k a, G_{R}$ varies as $(k a)^{1 / 2}$.

Combining Eqs. (6), (7), (9), (11), (13), and (14) the approximate ray form function is

$$
\begin{aligned}
f_{\mathrm{SWT}}^{(\infty)} \simeq & \mathscr{R} e^{-i 2 k a}-8 \sqrt{\pi k a} \beta_{R}^{\prime}(\infty) e^{i \Phi_{R}} \\
& \times \exp \left[-2\left(\pi-\theta_{R}\right) k a \beta_{R}^{\prime}(\infty)\right] \\
& \times \exp \left\{i k a\left[\left(c / c_{R}\right)\left(2 \pi-2 \theta_{R}\right)-2 \cos \theta_{R}\right]\right\} \\
& \times \sum_{m=0}^{\infty} e^{-2 \pi m k a \beta_{R}^{\prime}(\infty)} e^{i 2 \pi m k a c / c_{R}} .
\end{aligned}
$$

\section{B. Deformed cylinder formulation: Infinite and finite length}

In a recent article, we derived an approximate expression describing the sčattering of sound by deformed finite length cylinders where the cross-sectional radius, material composition profile (composition as a function of radial position), and bend of the axis were allowed to vary along the lengthwise axis of the cylinder. ${ }^{10}$ The orientation of the axis, plane-wave source direction, and point receiver position can also vary so long as the directions of incident and scattered fields are nearly perpendicular to the tangent of each point of the axis (this restriction can be relaxed under some conditions). The calculation involved integrating the volume flow per unit length of the cylinder. Since the expression for the volume flow was based on boundary conditions from the infinitely long cylinder, the boundary conditions of the de- 
formed cylinder are then assumed to vary slowly (i.e., the radius of curvature of the axial variations are large so that the solution to each slice of the cylinder is similar to that of an infinitely long cylinder of the same cylindrical radius). The solution was shown to be very accurate when describing high aspect ratio objects, where the aspect ratio is defined as the ratio of the length to diameter. Example calculations in that article involved the straight finite length cylinder, uniformly bent finite cylinder, and prolate spheroid.

While the emphasis of that article involved describing the scattering by deformed finite length cylinders, a more general solution was derived at the beginning that could describe the scattering by deformed cylinders of any length. That solution was then simplified for finite length cylinders to help facilitate calculations. (In that article, a cylinder is considered to be finite when the distance between its ends, $D_{e}$, is much smaller than the diameter of the first Fresnel zone $2 \sqrt{r \lambda}$ of the sonar transceiver. An infinite cylinder would span all of the Fresnel zones).

The general solution describing the scattering of sound by deformed cylinders of any length (including infinite) was expressed explicitly in terms of the modal series in Eq. (5) of Ref. 10. To facilitate use of that formula with both modal series and SWT representations, we rewrite the equation in terms of the form function:

$$
\begin{aligned}
p_{\text {scat }} \simeq & P_{0}\left(\frac{-i}{2 \sqrt{\pi}}\right) e^{i \pi / 4} \\
& \times \int_{r_{p o s}} f^{(\infty)} \sqrt{K a} \frac{e^{i k\left(r_{s}+\epsilon_{s}\right)}}{r_{s}}\left|d r_{p o s}\right| \text { (any length), }
\end{aligned}
$$

where $k r \gg 1$ and the wave numbers $K=k \sin \theta$ are shown explicitly in the integrand as well as implicitly in $f^{(\infty)}$. Note that although certain forms of $f^{(\infty)}$ may be exact (modal series representation ), $p_{\text {scat }}$ is generally approximate due to the assumptions given above.

The radius and cross-sectional profile of material properties are allowed to vary with $\mathbf{r}_{\text {pos }}$ and the details of the derivation of Eq. (16) (in modal series form) and associated figures describing scattering geometry can be found in Ref. 10. Note that the cylinder does not necessarily pass through the origin. Also, the cylinder can be of any length with respect to the diameter of the Fresnel zone provided, as stated above, the aspect ratio remains high. Finally, it is worth noting that while the above equation is used to provide approximate results when describing the scattering by finite length cylinders, it can be used to describe the scattering by an infinitely long undeformed cylinder exactly as it reproduces the well-known far-field solution given in Eq. (2). Equation (16) will be used in this article to describe the scattering by randomly rough infinitely long cylinders (once the roughness is introduced, the calculation becomes approximate).

While Eq. (16) is quite general, it can be simplified to describe typical geometries for finite length cylinders when the receiver is far away from the cylinder so that $r \gg D_{e}$ and, as mentioned above, $D_{e} \ll 2 \sqrt{r \lambda}$. Since the scattered field for finite cylinders spreads spherically as opposed to cylindrically in the infinitely long case, it was written in Ref. 10 in terms of the scattering amplitude $f_{\mathrm{DFC}}$ (deformed finite cylinder) as

$$
p_{\text {scat }}=P_{0}\left(e^{i k r} / r\right) f_{\mathrm{DFC}},
$$

where we rewrite $f_{\mathrm{DFC}}$ in terms of the form function as

$$
\begin{aligned}
f_{\mathrm{DFC}} \simeq & \frac{-i}{2 \sqrt{\pi}} e^{i \pi / 4} \int_{r_{\mathrm{pos}}} f^{(\infty)} \sqrt{K a} \\
& \times \exp \left[i k r_{\mathrm{pos}}\left(\hat{r}_{i}-\hat{r}_{r}\right) \cdot \hat{r}_{\text {pos }}\right]\left|d \mathbf{r}_{\text {pos }}\right| \quad \text { (finite length). }
\end{aligned}
$$

The cylinder is now far enough away so that it does not matter from which point on the cylinder the distance $r$ is measured. The reference phase depends upon the choice of the origin for $\mathbf{r}_{\text {pos }}$.

For the simple case of broadside incidence backscatter due to a (straight) finite length object with rotational symmetry about its lengthwise axis, the dot product in the exponent of Eq. (17) becomes zero and the above equation simplifies to

$$
f_{\mathrm{DFC}}=\frac{-i}{2 \sqrt{\pi}} e^{i \pi / 4} \int_{-L / 2}^{L / 2} f^{(\infty)} \sqrt{k a} d z
$$

(straight finite length, rotationally symmetric, broadside incidence backscatter) where the axis of the object now lies along the $z$ axis of the coordinate system and the wave number $K$ in the integrand becomes equal to $k$ as $\theta=90^{\circ}$. This equation is useful for objects such as the finite length cylinder and prolate spheroid (aspect ratio $\gtrsim 5: 1$ ) at broadside incidence.

We have thus rewritten previously derived integral formulas describing the scattering by deformed elongated objects in a general form so that either the modal series or Sommerfeld-Watson transformed solutions can be used in the form function $f^{(\infty)}$. This integral representation is useful as the scattered field per unit length of the object is integrated over its length. As shown in the next section, each infinitesimal slice of the object can have a radius with a random value as well as have correlation with nearby elements.

\section{Randomly rough elongated objects}

The roughness of the deformed cylinders is modeled as a perturbation, $\zeta\left(r_{\text {pas }}\right)$, of the radius about the mean radius

RANOOMLY ROUGH OEFORMED CYLINDER

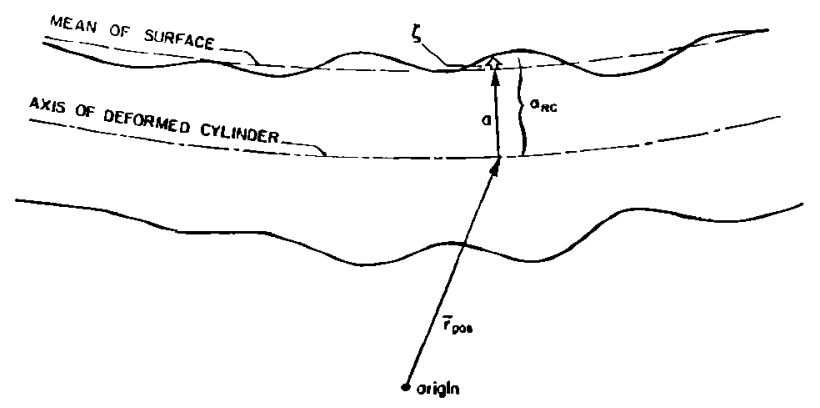

FIG. 2. General rough cylinder geometry illustrating mean ( $a$ ) and random ( $\zeta$ ) components of radius $a_{\mathrm{RC}}$. 
$a\left(\mathbf{r}_{\text {pos }}\right)$ so that the radius of the rough deformed cylinder is given as (Fig. 2)

$$
a_{\mathrm{RC}}\left(\mathbf{r}_{\mathrm{pos}}\right)=a\left(\mathbf{r}_{\mathrm{pos}}\right)+\zeta\left(\mathbf{r}_{\mathrm{pos}}\right) \text {. }
$$

The parameter $\xi\left(r_{\text {pos }}\right)$ is a stochastic variable with a zero mean such that

$$
\begin{aligned}
& \left\langle\xi\left(\mathbf{r}_{\mathrm{pos}}\right)\right\rangle=0, \\
& \left\langle a_{\mathrm{RC}}\left(\mathbf{r}_{\mathrm{pos}}\right)\right\rangle=a\left(\mathbf{r}_{\mathrm{pos}}\right) .
\end{aligned}
$$

The above equations describe roughness for cylinders of general deformed shape. That is, the roughness can be considered as a fine "texture" of cylinders that are also deformed on a larger scale. For example, the roughness can be superimposed upon straight cylinders or uniformly bent finite cylinders whose radius would have otherwise been constant with respect to position along the axis as well as prolate spheroids whose cross-sectional radius does vary along the axis.

For straight undeformed cylinders whose radius is constant in the zero roughness case, Eqs. (19) and (20) simplify to

$$
\begin{aligned}
& a_{\mathrm{RC}}(z)=a+\zeta(z), \\
& \left\langle a_{\mathrm{RC}}(z)\right\rangle=a .
\end{aligned}
$$

One of the above two formulas for $a_{\mathrm{RC}}$ will be incorporated into each of the previously derived scattering equations [Eqs. (16) $-(18)$ ] to describe the scattering by randomly rough deformed cylinders.

\section{EXPLICIT EXPRESSIONS}

\section{A. Straight finite cylinder: $k a \ll 1$}

\section{Mean scattering amplitude}

The general (far-field) scattering amplitude of the normal incidence backscattered field due to a straight finite length cylinder can be derived from Eqs. (4) and (18) as

$f_{\mathrm{DFC}} \simeq-\frac{i}{\pi} \int_{-L / 2}^{L / 2} \sum_{m=0}^{\infty} b_{m}(-1)^{m} d z \quad$ (straight cylinder)

where the axis of the cylinder is the $z$ axis.

For $k a \ll 1$, the modal summation for normal incidence backscatter due to a fluid cylinder is ${ }^{10}$

$$
\sum_{m=0}^{\infty} b_{m}(-1)^{m} \rightarrow \frac{i \pi}{2}(k a)^{2} \alpha_{\pi}, \quad k a \ll 1
$$

where

$$
\alpha_{\pi}=\frac{1-g h^{2}}{2 g h^{2}}+\frac{1-g}{1+g} .
$$

At these low frequencies, cylinders exhibit monopole(first term of $\alpha_{\pi}$ ) and dipole-like (second term of $\alpha_{\pi}$ ) behavior, hence the shear elastic properties are not a factor for elastic cylinders and they produce the same backscattering levels as fluid cylinders. " The following analysis therefore applies to both rough elastic and fluid cylinders in the $k a \ll 1$ region.

Applying the low-frequency limit of the modal summation given in Eq. (23) to Eq. (22) gives
FINITE LENGTH ROUGH CYLINDER $(L \ll 2 \sqrt{r \lambda})$

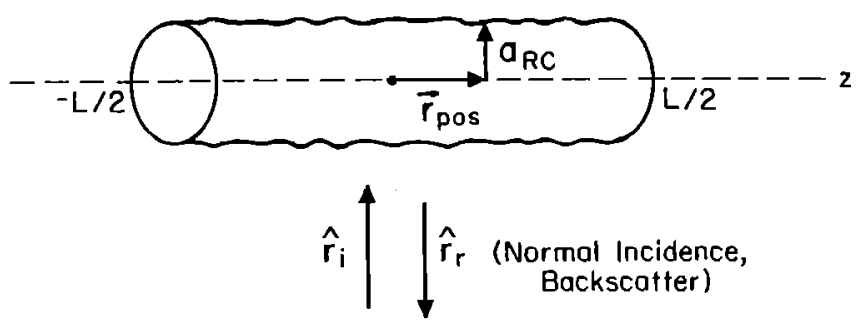

FIG. 3. Rough straight finite cylinder.

$$
f_{\mathrm{DFC}} \rightarrow \frac{1}{2} \alpha_{\pi} \int_{-L / 2}^{L / 2}(k a)^{2} d z, \quad k a \ll 1 .
$$

Randomizing the radius of the cylinder by setting $a_{\mathrm{RC}}=a+\zeta(z)$ [Eq. (21a)] we obtain the general low $k a$ formula for normal incidence backscattering due to a straight rough finite cylinder (Fig. 3):

$$
\begin{aligned}
f_{\mathrm{RC}} & =\frac{1}{2} \alpha_{\pi} \int_{-L / 2}^{L / 2}[k(a+\xi)]^{2} d z \\
& =\frac{1}{2} \alpha_{\pi}(k a)^{2} L+\frac{1}{2} \alpha_{\pi} \int_{-L / 2}^{L / 2}(k \xi)^{2} d z,
\end{aligned}
$$

where the first term of Eq. (25b) is equal to the scattering amplitude when the cylinder is perfectly smooth ${ }^{9}$ and the condition $\int_{-L / 2}^{L / 2} \zeta d z=0$ was used (zero mean roughness) on another term.

Assuming the roughness is Gaussian distributed with its PDF given in Eq. (A1) of Appendix A and using $\left\langle\xi^{2}\right\rangle=\sigma^{2}$ from Eq. (A5), the statistical mean of the scattering amplitude is found to be

$$
\begin{aligned}
\left\langle f_{\mathrm{RC}}\right\rangle & =\frac{1}{2} \alpha_{\pi}(k a)^{2} L\left[1+(\sigma / a)^{2}\right] \\
& =f_{0}\left[1+(\sigma / a)^{2}\right] .
\end{aligned}
$$

\section{Mean-square scattering amplitude}

From Eq. (25a), the square of the scattering amplitude of the rough cylinder is

$$
\begin{aligned}
f_{\mathrm{RC}}^{2}= & \frac{1}{4} \alpha_{\pi}^{2} k^{4} \int_{-L / 2}^{L / 2} \int_{-L / 2}^{L / 2}\left[a^{4}+2 a^{3} \xi\left(z^{\prime \prime}\right)+a^{2} \zeta^{2}\left(z^{\prime \prime}\right)\right. \\
& +2 a^{3} \zeta\left(z^{\prime}\right)+4 a^{2} \zeta\left(z^{\prime}\right) \zeta\left(z^{\prime \prime}\right)+2 a \xi\left(z^{\prime}\right) \zeta^{2}\left(z^{\prime \prime}\right) \\
& \left.+a^{2} \zeta^{2}\left(z^{\prime}\right)+2 a \zeta^{2}\left(z^{\prime}\right) \zeta\left(z^{\prime \prime}\right)+\zeta^{2}\left(z^{\prime}\right) \zeta^{2}\left(z^{\prime \prime}\right)\right] d z^{\prime} d z^{\prime \prime}
\end{aligned}
$$

where, again, this involves normal incidence backscatter for $k a \ll 1$. The statistical mean of this expression involves the bivariate Gaussian PDF, $\mathscr{W}_{1,2}$, as given in Eq. (A2) of Appendix $\mathrm{A}$. The terms in the brackets in the integrand of Eq. (27) involve various combinations of $\zeta\left(z^{\prime}\right), \zeta\left(z^{\prime \prime}\right)$, and their products, resulting in six unique classes of integrals. The integrals (means) are summarized in Eqs. (A3)-(A8) of Appendix A where the short hand notations $\zeta^{\prime} \equiv \zeta\left(z^{\prime}\right)$ and $\zeta^{\prime \prime} \equiv \zeta\left(z^{\prime \prime}\right)$ were used. Using those integrals to calculate the mean of $f^{2}$ in $\mathrm{Eq}$. (27) gives us the final result 


$$
\begin{aligned}
\left(f_{\mathrm{RC}}^{2}\right)= & f_{0}^{2}\left[1+2\left(\frac{\sigma}{a}\right)^{2}+\left(\frac{\sigma}{a}\right)^{4}\right. \\
& \left.\times\left(1+2 L^{-2} \int_{-L / 2}^{L / 2} \int_{-L / 2}^{L / 2} \Phi^{2}(\xi) d z^{\prime} d z^{\prime \prime}\right)\right],
\end{aligned}
$$

where the remaining integrals involve the autocorrelation function $\Phi(\xi)$ of the surface. Equation (28) cannot be evaluated without knowing the autocorrelation function.

By assuming a simple autocorrelation function illustrated in Appendix B that decreases linearly with $\xi$ in the range $0 \leqslant|\xi| \leqslant \mathscr{L}$ beyond which it remains zero for $|\xi|>\mathscr{L}$, it was shown in that appendix that the integral in Eq. (28) could be evaluated. Using the results in Appendix B, Eq. (28) becomes approximately

$$
\left\langle f_{\mathrm{RC}}^{2}\right\rangle \simeq f_{0}^{2}\left[1+2\left(\frac{\sigma}{a}\right)^{2}+\left(\frac{\sigma}{a}\right)^{4}\left(1+\frac{4}{3} \frac{\mathscr{L}}{L}\right)\right],
$$

where the approximate form of the integral was used $(\mathscr{L} / L \ll 1)$.

\section{B. Straight finite cylinder: ka $\gg 1$ \\ 1. Mean scattering amplitude}

As in the analysis in the previous section where $k a \ll 1$, we begin with Eq. (18). Rather than using the modal-seriesbased form function, we now apply the SWT-based form function $f_{\text {SwT }}^{(\infty)}$ given in Eq. (15). Randomizing the radius by inserting Eq. (21a) into $f_{\mathrm{SWT}}^{(\infty)}$ and inserting the resultant randomized form function into Eq. (18), we obtain

$$
\begin{aligned}
f_{\mathrm{RC}}= & \frac{-i}{2 \sqrt{\pi}} e^{i \pi / 4} \int_{-L / 2}^{L / 2}\left\{\mathscr{R} e^{-i 2 k \mid a+\zeta(z))} \sqrt{k[a+\zeta(z)]}-8 \sqrt{\pi} k[a+\zeta(z)] \beta_{R}^{\prime}(\infty) e^{i \Phi_{R}}\right. \\
& \times \exp \left\{-2\left(\pi-\theta_{R}\right) k[a+\zeta(z)] \beta_{R}^{\prime}(\infty)\right\} \exp \left\{i k[a+\zeta(z)]\left[\left(c / c_{R}\right)\left(2 \pi-2 \theta_{R}\right)-2 \cos \theta_{R}\right]\right\} \\
& \times \sum_{m=0}^{\infty} \exp \left\{-2 \pi m k[a+\zeta(z)] \beta_{R}^{\prime}(\infty)\right\} \exp \left\{i 2 \pi m k[a+\zeta(z)] c / c_{R}\right\} d z .
\end{aligned}
$$

This equation expresses the scattered field explicitly in terms of both phase and amplitude variations of rays from infinitesimal sections of the cylinder at point $z$ of length $d z$ that are perturbed by the random variable $\zeta(z)$. The total scattered field due to the entire cylinder will be the sum of the infinitesimal contributions. Since $\zeta(z)$ is a stochastic variable, the total scattered field will fluctuate from realization to realization of the surface. In order to describe statistical averages of the scattered field in a simple analytical manner, further simplifications must be made.

Appendix $C$ shows that the contributions of the complex or phase-shift terms $e^{-i 2 k \zeta}$ and $\exp \left\{i k \xi\left[\left(c / c_{R}\right)(2 \pi\right.\right.$ $\left.\left.\left.-2 \theta_{R}\right)-2 \cos \theta_{R}+2 \pi m c / c_{R}\right]\right\}$ dominate the dependence of the mean and mean-square terms upon roughness while the amplitude-variation terms $\sqrt{k(a+\zeta)}, k(a+\zeta)$, $\exp \left[-2\left(\pi-\theta_{R}\right) k \zeta \beta_{R}^{\prime}(\infty)\right]$, and $\exp \left[-2 \pi m k \zeta \beta_{R}^{\prime}(\infty)\right]$ influence the averages a negligible amount. We therefore neglect $\zeta(z)$ in the (amplitude variation) real factors to each term in the integrand while keeping $\zeta(z)$ in the (phase-shift) complex exponents. We now write the mean of Eq. (30) as

$$
\begin{aligned}
\left\langle f_{\mathrm{RC}}\right\rangle= & \frac{-i}{2 \sqrt{\pi}} e^{i \pi / 4}\left\langle\int _ { - L / 2 } ^ { L / 2 } \left\{\mathscr{R} e^{-i 2 k a} e^{i 2 k \zeta(z)} \sqrt{k a}-8 \sqrt{\pi} k a \beta \beta_{R}^{\prime}(\infty) e^{i \Phi_{R}}\right.\right. \\
& \times \exp \left[-2\left(\pi-\theta_{R}\right) k a \beta_{R}^{\prime}(\infty)\right] \exp \left(i k a\left[\left(c / c_{R}\right)\left(2 \pi-2 \theta_{R}\right)-2 \cos \theta_{R}\right]\right\} \sum_{m=0}^{\infty} \exp \left[-2 \pi m k a \beta_{R}^{\prime}(\infty)\right] \\
& \left.\times \exp \left(i 2 \pi m k a c / c_{R}\right) \exp \left\{i k \zeta(z)\left[\left(c / c_{R}\right)\left(2 \pi-2 \theta_{R}\right)-2 \cos \theta_{R}+2 \pi m c / c_{R}\right]\right\} d z\right)
\end{aligned}
$$

where only the $\zeta$ terms that dominate the mean and meansquare remain. Note that although the terms ignored in Eq. (31) contribute a negligible amount to the means, they may be important when describing the statistics of the echo envelope, hence the more general Eq. (30) would be the equation to use in that case. ${ }^{55}$

Equation (31) is in a form that the mean of the backscattered echo due to the rough cylinder can be evaluated and interpreted in a straightforward manner. The variations in the integrand due to the roughness show up solely in the exponents and represent fluctuations in the phase of the respective terms. This formula is broadly similar in form to the Helmholtz-Kirchhoff surface integral where the differential Huygen wavelets are summed over the entire surface to produce the total scattered field. ${ }^{56}$ For far-field normal inci- dence backscatter due to a rough surface and small-slope conditions that allow one to invoke the Kirchhoff approximation, the perturbations of the wavelets due to the roughness show up simply as variations in their phase. Eckart and many later investigators studied the statistical properties of echoes from randomly rough planar interfaces ${ }^{46-54}$ and the following analysis involving rough cylinders will be performed in an analogous manner. One difference between this analysis and some rough (planar) surface scattering theories is that the cylinder is taken to be rough only in one dimension where many of the rough planar surfaces are two dimensional.

Since $\zeta(z)$ is a stochastic parameter, the mean of the terms in the integrand must be calculated before the integral over $z$ is performed. Equation (31) is then rewritten slightly: 


$$
\begin{aligned}
\left\langle f_{\mathrm{RC}}\right\rangle= & \frac{-i}{2 \sqrt{\pi}} e^{i \pi / 4} \int_{-L / 2}^{L / 2}\left\{\mathscr{R} e^{-i 2 k a} \sqrt{k a}\left\langle e^{-i 2 k \zeta(z)}\right\rangle-8 \sqrt{\pi} k a \beta_{R}^{\prime}(\infty) e^{i \Phi_{R}}\right. \\
& \times \exp \left[-2\left(\pi-\theta_{R}\right) k a \beta_{R}^{\prime}(\infty)\right] \exp \left\{i k a\left[\left(c / c_{R}\right)\left(2 \pi-2 \theta_{R}\right)-2 \cos \theta_{R}\right]\right\} \sum_{m=0}^{\infty} \exp \left[-2 \pi m k a \beta_{R}^{\prime}(\infty)\right] \\
& \left.\times \exp \left(i 2 \pi m k a c / c_{R}\right)\left\langle\exp \left\{i k \zeta(z)\left[\left(c / c_{R}\right)\left(2 \pi-2 \theta_{R}\right)-2 \cos \theta_{R}+2 \pi m c / c_{R}\right]\right\}\right\rangle\right\} d z
\end{aligned}
$$

Evaluation of this equation involves knowledge of the distribution of $\xi(z)$. For rough planar interface calculations, it is mathematically convenient and physically reasonable to assume that the roughness follows Gaussian statistics. ${ }^{50}$ For the same reasons, we assume the distribution of the roughness of the cylinders to be Gaussian as described by Eq. (A1) in Appendix A. Using that formula for $W(\zeta)$, it can be shown that ${ }^{46}$

$$
\left\langle e^{i a \zeta}\right\rangle=e^{-(1 / 2) a^{2} \sigma^{2}} \text {. }
$$

Applying Eq. (33) to the mean terms in Eq. (32) we obtain

$$
\left\langle e^{-i 2 k 5}\right\rangle=e^{-2 k^{2} \sigma^{2}}
$$

and

$$
\begin{aligned}
& \left\langle\exp \left\{i k\left[\left(c / c_{R}\right)\left(2 \pi-2 \theta_{R}\right)-2 \cos \theta_{R}+2 \pi m c / c_{R}\right] \xi\right\}\right\rangle \\
& \quad=\exp \left\{(-1 / 2) k^{2} \sigma^{2}\left[\left(c / c_{R}\right)\left(2 \pi \cdot-2 \theta_{R}\right)-2 \cos \theta_{R}+2 \pi m c / c_{R}\right]^{2}\right\} .
\end{aligned}
$$

Inserting Eqs. (34) and (35) into Eq. (32) and performing what is now a trivial integration over $z$, Eq. (32) becomes

$$
\begin{aligned}
\left\langle f_{\mathrm{RC}}\right\rangle= & (-i / 2 \sqrt{\pi}) e^{i \pi / 4} \mathscr{R} \sqrt{k a} L e^{-i 2 k a} e^{-2 k^{2} \sigma^{2}}+4 i e^{i \pi / 4} k a L \beta_{R}^{\prime}(\infty) e^{i \Phi_{R}} \\
& \times \exp \left[-2\left(\pi-\theta_{R}\right) k a \beta_{R}^{\prime}(\infty)\right] \exp \left\{i k a\left[\left(c / c_{R}\right)\left(2 \pi-2 \theta_{R}\right)-2 \cos \theta_{R}\right]\right\} \sum_{m=0}^{\infty} \exp \left[-2 \pi m k a \beta_{R}^{\prime}(\infty)\right] \\
& \times \exp \left(i 2 \pi m k a c / c_{R}\right) \exp \left\{-(1 / 2) k^{2} \sigma^{2}\left[\left(c / c_{R}\right)\left(2 \pi-2 \theta_{R}\right)-2 \cos \theta_{R}+2 \pi m c / c_{R}\right]^{2}\right\} .
\end{aligned}
$$

\section{Mean square scattering amplitude}

Because of the large size of the cross terms that result from taking the mean square of the field, we make the following definitions:

$$
\begin{aligned}
& C_{R} \equiv\left(c / c_{R}\right)\left(2 \pi-2 \theta_{R}\right)-2 \cos \theta_{R} \\
& g_{R}^{(1)} \equiv \beta_{R}^{\prime}(\infty) e^{i \Phi_{R}} \exp \left[-2\left(\pi-\theta_{R}\right) k a \beta_{R}^{\prime}(\infty)\right] e^{i k a C_{R}} \\
& g_{R}^{(2)}(m) \equiv \exp \left[-2 \pi m k a \beta_{R}^{\prime}(\infty)\right] \exp \left(i 2 \pi m k a c / c_{R}\right)
\end{aligned}
$$

Inserting these terms into Eq. (30), taking the mean square of the field, and ignoring the amplitude variation terms as justified in Appendix C:

$$
\begin{aligned}
\left\langle f_{\mathrm{RC}} f_{\mathrm{RC}}^{*}\right\rangle= & \frac{1}{4 \pi} \int_{-L / 2}^{L / 2} \int_{-L / 2}^{L / 2}\left\{\mathscr{R}^{2} k a\left\langle\exp \left\{i 2 k\left[\zeta\left(z^{\prime \prime}\right)-\zeta\left(z^{\prime}\right)\right]\right\}\right\rangle-\mathscr{R} e^{-i 2 k a}(k a)^{3 / 2} 8 \sqrt{\pi}\left(g_{R}^{(1)}\right)^{*}\right. \\
& \times \sum_{m^{\prime}=0}^{\infty}\left[g_{R}^{(2)}\left(m^{\prime \prime}\right)\right]^{*}\left\langle\exp \left(-i k\left\{2 \zeta\left(z^{\prime}\right)+\left(C_{R}+2 \pi m^{\prime \prime} c / c_{R}\right) \xi\left(z^{\prime \prime}\right)\right\}\right)\right\rangle-\text { c.c. }+64 \pi k^{2} a^{2}\left|g_{R}^{(1)}\right|^{2} \\
& \times \sum_{m^{\prime}=0}^{\infty} \sum_{m^{\prime \prime}=0}^{\infty} g_{R}^{(2)}\left(m^{\prime}\right)\left[g_{R}^{(2)}\left(m^{\prime \prime}\right)\right]^{*}\left\langle\exp \left\{i k\left[\left(C_{R}+2 \pi m^{\prime} c / c_{R}\right) \zeta\left(z^{\prime}\right)-\left(C_{R}+2 \pi m^{\prime \prime} c / c_{R}\right) \zeta\left(z^{\prime \prime}\right)\right]\right\rangle\right\} d z^{\prime} d z^{\prime \prime}
\end{aligned}
$$

where the primes and double primes correspond to the $z^{\prime}$ and $z^{\prime \prime}$ integrals, respectively, and c.c. stands for complex conjugate.

Each term in Eq. (40) has a mean of similar form. The values of $\zeta\left(z^{\prime}\right)$ and $\xi\left(z^{\prime \prime}\right)$ are interdependent and require use of a bivariate distribution to describe their statistical properties. Assuming the roughness variable $\xi$ [upon which $\xi\left(z^{\prime}\right)$ and $\xi\left(z^{\prime \prime}\right)$ are based] is Gaussian distributed, the bivariate Gaussian distribution $\mathscr{W}_{1,2}$ as described in Appendix A is used to calculate the averages in Eq. (40). The solution to the above averages is given in the following general formula:

$$
\left\langle e^{i\left(A \xi^{\prime}+B \xi^{\prime \prime}\right)}\right\rangle=\exp \left\{-\left(\sigma^{2} / 2\right)\left[A^{2}+2 A B \Phi(\xi)+B^{2}\right]\right\} .
$$

The details of the derivation of Eq. (41) are similar to those discussed in the aforementioned surface roughness articles (where $A=-B=1$ ) and will not be repeated here. Applying Eq. (41) to the appropriate terms in Eq. (40), we obtain 


$$
\begin{aligned}
\left\langle f_{\mathrm{RC}} f_{\mathrm{RC}}^{*}\right\rangle= & \frac{1}{4 \pi} \int_{-L / 2}^{L / 2} \int_{-L / 2}^{L / 2}\left[\mathscr{R}^{2} k a \exp \left\{-4 k^{2} \sigma^{2}[1-\Phi(\xi)]\right\}-\mathscr{R} e^{-i 2 k a}(k a)^{3 / 2} 8 \sqrt{\pi}\left(g_{R}^{(1)}\right)^{*} \sum_{m^{\prime}=0}^{\infty}\left[g_{R}^{(2)}\left(m^{\prime \prime}\right)\right]^{*}\right. \\
& \times \exp \left(-4 k^{2} \sigma^{2}\left\{\frac{1}{2}+\frac{1}{8}\left(2 \pi m^{\prime \prime} c / c_{R}+C_{R}\right)^{2}+\frac{1}{2}\left(2 \pi m^{\prime \prime} c / c_{R}+C_{R}\right) \Phi(\xi)\right\}\right)-c . c .+64 \pi k^{2} a^{2}\left|g_{R}^{(1)}\right|^{2} \\
& \times \sum_{m^{\prime}=0}^{\infty} \sum_{m^{\prime}=0}^{\infty} g_{R}^{(2)}\left(m^{\prime}\right)\left[g_{R}^{(2)}\left(m^{\prime \prime}\right)\right]^{*} \exp \left(-4 k^{2} \sigma^{2}\left\{\frac{1}{8}\left(2 \pi m^{\prime} c / c_{R}+C_{R}\right)^{2}+\frac{1}{8}\left(2 \pi m^{\prime \prime} c / c_{R}+C_{R}\right)^{2}\right.\right. \\
& \left.\left.\left.-\frac{1}{4}\left(2 \pi m^{\prime} c / c_{R}+C_{R}\right)\left(2 \pi m^{\prime \prime} c / c_{R}+C_{R}\right) \Phi(\xi)\right\}\right)\right\} d z^{\prime} d z^{\prime \prime} .
\end{aligned}
$$

The terms to be integrated in Eq. (42) all have the form $\iint F[\Phi(\xi)] d z^{\prime} d z^{\prime \prime}$. Condensing the equation, we obtain

$$
\begin{aligned}
\left\langle f_{\mathrm{RC}} f_{\mathrm{RC}}^{*}\right\rangle= & \frac{1}{4 \pi} \mathscr{R}^{2} k a e^{-4 k^{2} \sigma^{2}} I(-1)-\mathscr{R} e^{-i 2 k a}(k a)^{3 / 2} 2 \pi^{-1 / 2}\left(g_{R}^{(1)}\right)^{*} \sum_{m^{\prime \prime}=0}^{\infty}\left[g_{R}^{(2)}\left(m^{\prime \prime}\right)\right]^{*} \\
& \times \exp \left\{-4 k^{2} \sigma^{2}\left[\frac{1}{2}+\frac{1}{8}\left(2 \pi m^{\prime \prime} c / c_{R}+C_{R}\right)^{2}\right]\right\} I\left[\frac{1}{2}\left(2 \pi m^{\prime \prime} c / c_{R}+C_{R}\right)\right]-\text { c.c. } \\
& +16 k^{2} a^{2}\left|g_{R}^{(1)}\right|^{2} \sum_{m^{\prime}=0}^{\infty} \sum_{m^{\prime}=0}^{\infty} g_{R}^{(2)}\left(m^{\prime}\right)\left[g_{R}^{(2)}\left(m^{\prime \prime}\right)\right]^{*} \\
& \times \exp \left\{-4 k^{2} \sigma^{2}\left[\frac{1}{8}\left(2 \pi m^{\prime} c / c_{R}+C_{R}\right)^{2}+\frac{1}{8}\left(2 \pi m^{\prime \prime} c / c_{R}+C_{R}\right)^{2}\right]\right\} \\
& \times I\left[-\frac{1}{4}\left(2 \pi m^{\prime} c / c_{R}+C_{R}\right)\left(2 \pi m^{\prime \prime} c / c_{R}+C_{R}\right)\right],
\end{aligned}
$$

where

$$
I(\alpha)=\int_{-L / 2}^{L / 2} \int_{-L / 2}^{L / 2} e^{-4 k^{2} \sigma^{2} \alpha \Phi(\xi)} d z^{\prime} d z^{\prime \prime}
$$

Equation (43) is quite complex and long. In the low $k \sigma$ limit, the equation simplifies greatly and becomes useful to examine. The reader is referred to Ref. 55 for an analysis in that region.

\section{Other shapes}

As demonstrated in Ref. 10, the deformed cylinder solution can be used to accurately describe the scattering of sound by other objects of high aspect ratio $(\gtrsim 5: 1)$ such as the prolate spheroid, uniformly bent finite cylinder, and infinitely long cylinder at or near broadside incidence. We now apply the previous analysis and the methods presented in Ref. 10 to describe the scattering by those objects with rough boundaries. For the sake of brevity, only the mean field in the geometric scattering region will be considered. In each case, only broadside incidence is investigated. form

In each of the following calculations, an integral of the

$$
\psi=\int_{\mathrm{C}}^{D} G(v) e^{i F(v)} d v
$$

will have to be solved where $G(v)$ varies slowly with respect to $F(v)$. Since in each case the condition $F^{\prime}(A)=0$ for $C<A<D$ exists, then the method of stationary phase can be applied to give the approximate general solution

$$
\psi \simeq \sqrt{\frac{2 \pi}{\left|F^{\prime \prime}(A)\right|}} G(A) \exp \left[i\left(F(A) \pm \frac{\pi}{4}\right)\right],
$$

where each prime on $F$ denotes the derivative with respect to its argument, and the "+" and "-" signs apply to $F^{\prime \prime}(A)>0$ and $F^{\prime \prime}(A)<0$, respectively. ${ }^{58}$ The solution assumes that the only value of $v$ at which $F^{\prime}$ is zero in the $C-D$ range is at $v=A$.
Also, for reasons similar to those given in Sec. II B and Appendix $\mathrm{C}$, the amplitude variation terms will be ignored in the calculation of the mean scattered field due to the following objects as the phase shift terms dominate the scattering.

\section{Prolate spheroid}

The prolate spheroid is considered in the deformed cylinder formulation to be essentially a long finite "cylinder" with its radius slowly decreasing to zero at the ends. While the cross-sectional radius of the spheroid varies, the rms roughness is assumed to be constant along the entire length of the object. Because we are dealing with high-aspect-ratio spheroids, most of the surface is nearly parallel with the ma-

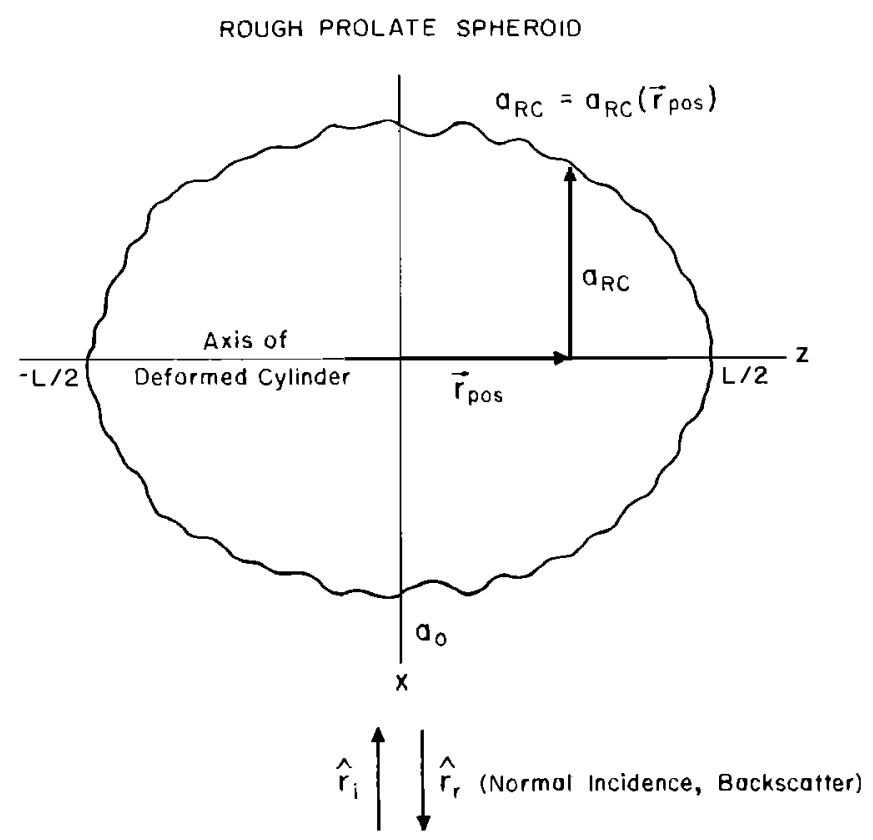

FIG. 4. Rough prolate spheroid.

T. K. Stanton: Scattering by rough objects. I. 


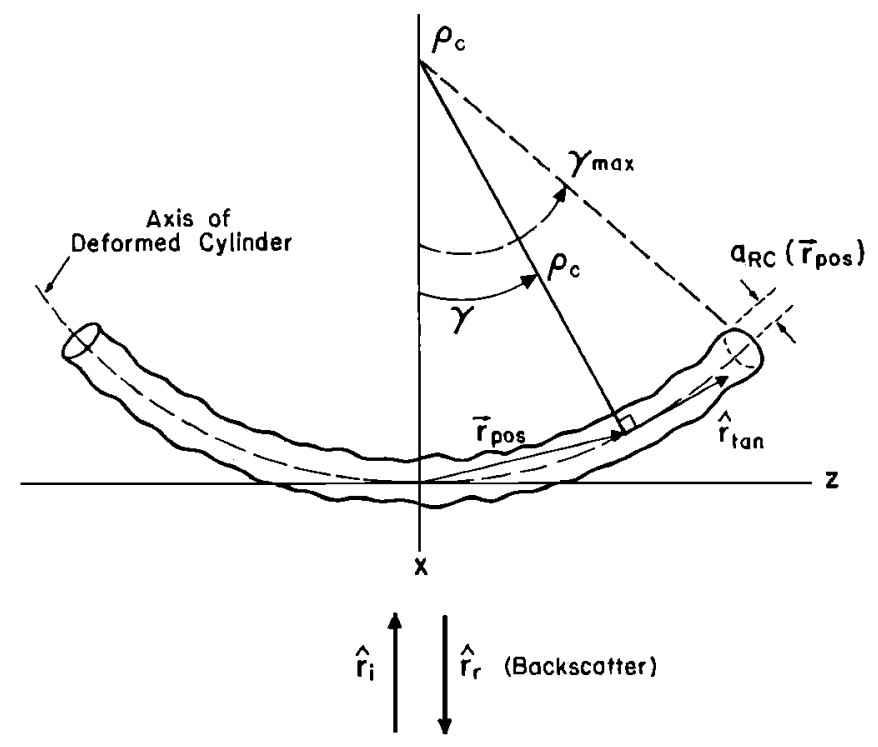

FIG. 5. Rough uniformly bent finite cylinder with radius of curvature $\rho_{c}$. The cylinder is shown to be bent away from the sonar.

jor axis (especially near the origin). For broadside incidence, as shown in Fig. 4, that section of the surface that is contained in the plane determined by both the incident wave vector and the major axis is then nearly perpendicular to the direction of incidence (again, especially near the origin).

In this "small-slope" case, the roughness is assumed to vary in a direction along the cylindrical radius. In the plane just mentioned, the roughness would vary in a direction parallel to the direction of the incident plane wave. Because of this assumption, the rough spheroid described mathematically possibly may not describe an actual rough spheroid near the ends. For a metal object machined by a lathe, if the cutting tool is applied only in the direction perpendicular to the major axis, then it is possible that any roughness due to imperfections in the cutting process would vary in the direction perpendicular to the major axis (i.e., radially as the model assumes). If, then, that object is sanded and polished by hand, the remaining microroughness would vary in a direction normal to the surface. Either way, because we are describing only high aspect ratio objects at broadside incidence, it is reasonable to ignore the scattering properties at and near the ends for the following (qualitative) reasons: (1) this "large-slope" region only takes up a small fraction of the object and hence its relative contribution would be proportionately smaller, (2) near the ends, the radius decreases to zero. As it approaches zero, the scattering cross section crosses into the Rayleigh scattering region $(k a \ll 1)$ and becomes negligibly small compared with the scattering from the mid-section of the object which remains in the geometric scattering region $\left(k a_{0} \gg 1\right)$, and $(3)$ in this $k a_{0} \gg 1$ case, the ends of the spheroid will be outside the first Fresnel zone. The higher-order zones containing the ends are of alternating phase and tend to cancel each other (and accompanying errors) out.

For broadside incidence and distances far from the spheroid so that $D_{e} \ll 2 \sqrt{r \lambda}$, we use Eq. (18) to describe the scattering. For simplicity, the center of the spheroid is at the origin with the major axis lying along the $z$ axis (see Fig. 4). The difference between this formulation and the one we used to describe the scattering by finite cylinders in Eq. (30) is that the mean radius varies along the length of the object giving it the shape of a spheroid. The radius is given by

$$
a_{\mathrm{RC}}(z)=a_{0} \sqrt{1-[z /(L / 2)]^{2}}+\zeta(z) \text {. }
$$

This equation was derived by setting $a\left(\mathbf{r}_{\mathrm{pos}}\right)$ in Eq. (19) equal to the equation for the radius of the smooth spheroid as described in Ref. 10 and, again, $r_{\text {pos }}$ becomes $z$. We now proceed in the same manner when the general scattering equations were derived for the rough straight finite cylinder [Eq. (30) ]. Inserting Eqs. (15) and (47) into Eq. (18), we obtain the scattering amplitude

$$
\begin{aligned}
f_{\mathrm{RC}}= & \frac{-i}{2 \sqrt{\pi}} e^{i \pi / 4} \int_{-L / 2}^{L / 2}\left\{\mathscr{R} \sqrt{k\left\{a_{0} \sqrt{1-[z /(L / 2)]^{2}}+\zeta(z)\right\}} \exp \left(-i 2 k\left\{a_{0} \sqrt{1-[z /(L / 2)]^{2}}+\zeta(z)\right\}\right)\right. \\
& -8 \sqrt{\pi} k\left[a_{0} \sqrt{1-[z /(L / 2)]^{2}}+\zeta(z)\right] \beta_{R}^{\prime}(\infty) e^{i \Phi_{R}} \exp \left(-2\left(\pi-\theta_{R}\right) k\left\{a_{0} \sqrt{1-[z /(L / 2)]^{2}}+\zeta(z)\right\} \beta_{R}^{\prime}(\infty)\right) \\
& \times \exp \left(i k\left\{a_{0} \sqrt{1-[z /(L / 2)]^{2}}+\zeta(z)\right\}\left[\left(c / c_{R}\right)\left(2 \pi-2 \theta_{R}\right)-2 \cos \theta_{R}\right]\right) \sum_{m=0}^{\infty} \exp (-2 \pi m k \\
& \left.\left.\times\left\{a_{0} \sqrt{1-[z /(L / 2)]^{2}}+\zeta(z)\right\} \beta_{R}^{\prime}(\infty)\right) \exp \left(i 2 \pi m k\left\{a_{0} \sqrt{1-[z /(L / 2)]^{2}}+\zeta(z)\right\} c / c_{R}\right)\right\} d z .
\end{aligned}
$$

Analysis using this equation is not valid near the ends where the cross-sectional radius approaches zero as the roughness perturbation becomes greater than the radius. But for the same reasons given earlier in this section, contributions to the scattering from near the ends are assumed to be negligible hence making errors in this region also negligible.

Ignoring all amplitude variation terms and making the substitutions $\mu=z /(L / 2)$ and $d \mu=d z /(L / 2)$, the stationary phase parameters are calculated with $F(\mu)=-2 k a_{0} \sqrt{1-\mu^{2}}$ and $G(\mu)=\left(1-\mu^{2}\right)^{1 / 4}$ for the first term. In the second term of Eq. (48), the parameters are

$$
\begin{aligned}
& F(\mu)=k a_{0} \sqrt{1-\mu^{2}}\left[\left(c / c_{R}\right)\left(2 \pi-2 \theta_{R}\right)-2 \cos \theta_{R}+2 \pi m c / c_{R}\right] \\
& G(\mu)=\sqrt{1-\mu^{2}} \exp \left[-2\left(\pi-\theta_{R}\right) k a_{0} \sqrt{1-\mu^{2}} \beta_{R}^{\prime}(\infty)\right] \exp \left[-2 \pi m k a_{0} \sqrt{1-\mu^{2}} \beta_{R}^{\prime}(\infty)\right] .
\end{aligned}
$$


The $F^{\prime}=0$ stationary point is at $\mu=0$ in each term. Using these stationary phase parameters in Eq. (46) gives the approximate high-frequency $(k a \gg 1)$ solution to the mean scattered field of the randomly rough prolate spheroid:

$$
\begin{aligned}
\left\langle f_{\mathrm{RC}}\right\rangle= & \frac{1}{4} \mathscr{R} L e^{-i 2 k a_{0}} e^{-2 k^{2} \sigma^{2}}+2 i \sqrt{k a_{0}} L \beta_{R}^{\prime}(\infty) \exp \left[-2\left(\pi-\theta_{R}\right) k a_{0} \beta_{R}^{\prime}(\infty)\right] e^{i \Phi_{R}} \\
& \times \sum_{m=0}^{\infty} \sqrt{\frac{2 \pi}{\left(c / c_{R}\right)\left(2 \pi-2 \theta_{R}\right)-2 \cos \theta_{R}+2 \pi m c / c_{R}}} \exp \left[-2 \pi m k a_{0} \beta_{R}^{\prime}(\infty)\right] \\
& \times \exp \left\{i k a_{0}\left[\left(c / c_{R}\right)\left(2 \pi-2 \theta_{R}\right)-2 \cos \theta_{R}+2 \pi m c / c_{R}\right]\right\} \\
& \times \exp \left\{-\frac{1}{2} k^{2} \sigma^{2}\left[\left(c / c_{R}\right)\left(2 \pi-2 \theta_{R}\right)-2 \cos \theta_{R}+2 \pi m c / c_{R}\right]^{2}\right\} .
\end{aligned}
$$

\section{Uniformly bent finite cyllinder}

The backscattered sound for this object is calculated for "broadside" incidence where the cylinder is bent symmetrically away from the source/receiver (Fig. 5). As with the other cases, the roughness will be a function of position along the axis and will vary radially from the axis. Similar to the straight cylinder and in contrast to the prolate spheroid, the radial variation will be normal to the mean surface.

In the case of the uniformly bent cylinder, we begin with the more general deformed finite cylinder formulation, Eq. (17). It was shown in Ref. 10 that the position vector $\mathbf{r}_{\mathrm{pos}}=\rho_{c}[(1-\cos \gamma) \hat{i}+\sin \gamma \hat{k}],\left|d \mathbf{r}_{\mathrm{pos}}\right|=\rho_{c} d \gamma$, and the exponent in Eq. (17) of this article becomes $2 i k \rho_{c}(1-\cos \gamma)$. Also, the wave numbers in the form function and square root factor are multiplied by the quantity $|\cos \gamma|$.

Substituting $\rho_{c} \gamma$ for $\mathbf{r}_{\text {pos }}$ in Eq. (19) gives an expression for the cross-sectional radius of the rough bent cylinder:

$$
a_{\mathrm{RC}}\left(\mathbf{r}_{\mathrm{pos}}\right)=a+\zeta\left(\rho_{c} \gamma\right),
$$

where the radius $a$ is a constant. Incorporating the bent cylinder geometry into Eqs. (15) and (17) gives the following general equation describing the backscattering by the rough bent cylinder:

$$
\begin{aligned}
f_{\mathrm{RC}}= & \frac{-i \rho_{c}}{2 \sqrt{\pi}} e^{i \pi / 4} \int_{-\gamma_{\max }}^{\gamma_{\max }}\left\{\mathscr{R} \sqrt{K\left[a+\zeta\left(\rho_{c} \gamma\right)\right]} \exp \left\{-i 2 K\left[a+\zeta\left(\rho_{c} \gamma\right)\right]\right\} \exp \left[2 i k \rho_{c}(1-\cos \gamma)\right]\right. \\
& -8 \sqrt{\pi} K\left[a+\zeta\left(\rho_{c} \gamma\right)\right] \beta_{R}^{\prime}(\infty) e^{i \Phi_{R}} \exp \left\{-2\left(\pi-\theta_{R}\right) K\left[a+\zeta\left(\rho_{c} \gamma\right)\right] \beta_{R}^{\prime}(\infty)\right\} \\
& \times \exp \left\{i K\left[a+\zeta\left(\rho_{c} \gamma\right)\right]\left[\left(c / c_{R}\right)\left(2 \pi-2 \theta_{R}\right)-2 \cos \theta_{R}\right]\right\} \sum_{m=0}^{\infty} \exp \left\{-2 \pi m K\left[a+\zeta\left(\rho_{c} \gamma\right)\right] \beta_{R}^{\prime}(\infty)\right\} \\
& \times \exp \left\{i 2 \pi m K\left[a+\zeta\left(\rho_{c} a\right)\right] c / c_{R}\right\} \exp \left[2 i k \rho_{c}(1-\cos \gamma)\right\} d \gamma,
\end{aligned}
$$

where the angle $2 \gamma_{\max }$ subtends the entire arc, the total arc length $L=2 \rho_{c} \gamma_{\max }$, and $K=k \cos \gamma$. Since the angle between directions of incidence and reception and the direction of the tangent to the axis must be nearly $90^{\circ}$, then $\gamma_{\max }$ cannot be too large, although under some circumstances the restriction can be relaxed. ${ }^{10}$

Ignoring the amplitude variation terms and applying the method of stationary phase, the stationary phase parameters for each term of the mean of the above equation are

First term:

$$
\begin{aligned}
& F(\gamma)=-2 k a \cos \gamma+2 k \rho_{c}(1-\cos \gamma), \\
& G(\gamma)=\sqrt{\cos \gamma} e^{-2 k^{2} \sigma^{2} \cos ^{2} \gamma .}
\end{aligned}
$$

Second term:

$$
\begin{aligned}
F(\gamma)= & k a \cos \gamma\left[\left(c / c_{R}\right)\left(2 \pi-2 \theta_{R}\right)-2 \cos \theta_{R}+2 \pi m c / c_{R}\right]+2 k \rho_{c}(1-\cos \gamma), \\
G(\gamma)= & \cos \gamma \exp \left[-2\left(\pi-\theta_{R}\right) k a \cos \gamma \beta_{R}^{\prime}(\infty)\right] \exp \left[-2 \pi m k a \cos \gamma \beta_{R}^{\prime}(\infty)\right] \\
& \times \exp \left\{-\frac{1}{2} k^{2} \sigma^{2} \cos ^{2} \gamma\left[\left(c / c_{R}\right)\left(2 \pi-2 \theta_{R}\right)-2 \cos \theta_{R}+2 \pi m c / c_{R}\right]^{2}\right\},
\end{aligned}
$$

where the $F^{\prime}=0$ stationary point is at $\gamma=0$ in each term.

Note that for typical values of $\rho_{c} / L \gtrsim 1$ and $k a \gtrsim 10, F^{\prime \prime}(0)$ in the second term is greater than zero for all multiply circumnavigated Rayleigh waves that do not experience substantial loss due to radiation damping hence we use the $+\pi / 4$ as prescribed in Eq. (46). Using the above stationary phase parameters in Eq. (46) gives the approximate high frequency $(k a \gg 1)$ solution to the mean scattered field of the randomly rough uniformly bent cylinder:

$$
\begin{aligned}
\left\langle f_{\mathrm{RC}}\right\rangle= & \frac{1}{2} \mathscr{R} \sqrt{\frac{\rho_{c} a}{1+a / \rho_{c}}} e^{-i 2 k a} e^{-2 k^{2} \sigma^{2}}-4 a \beta_{R}^{\prime}(\infty) \exp \left[-2\left(\pi-\theta_{R}\right) k a \beta_{R}^{\prime}(\infty) e^{i \Phi_{R}}\right] \\
& \times \sum_{m=0}^{\infty} \sqrt{\frac{\pi k \rho_{c}}{1-\left(a / 2 p_{c}\right)\left[\left(c / c_{R}\right)\left(2 \pi-2 \theta_{R}\right)-2 \cos \theta_{R}+2 \pi m c / c_{R}\right]}}
\end{aligned}
$$




$$
\begin{aligned}
& \times \exp \left\{-2 \pi m k a \beta_{R}^{\prime}(\infty)\right\} \exp \left\{i k a\left[\left(c / c_{R}\right)\left(2 \pi-2 \theta_{R}\right)-2 \cos \theta_{R}+2 \pi m c / c_{R}\right]\right\} \\
& \times \exp \left\{-\frac{1}{2} k^{2} \sigma^{2}\left[\left(c / c_{R}\right)\left(2 \pi-2 \theta_{R}\right)-2 \cos \theta_{R}+2 \pi m c / c_{R}\right]^{2}\right\}
\end{aligned}
$$

\section{Infinitely long, straight cylinder}

The solution to the infinitely long straight rough cylinder (Fig. 6) requires the more general scattering formula given in Eq. (16). In contrast to the finite-length objects, where the range to the cylinder $r$ is much greater than the length of the object, the distance $r_{s}$ to the individual slices of the cylinder as well as the phase of the resultant scattered field from each slice (even for a smooth cylinder) varies with respect to position along the cylinder. Thus Eq. (16) must be used to take both of these dependences into account. The solution is in the $k a \gg 1$ and $k r \gg 1$ region.

For normal incidence backscatter and the axis containing the origin, $\epsilon_{s}$ is zero in Eq. (16) and we integrate over the entire length of the cylinder. Inserting Eqs. (15) and (21a) into Eq. (16) gives the following equation for the backscattering from the infinitely long rough cylinder:

$$
\begin{aligned}
p_{\text {scat }}= & P_{0}\left(\frac{-i}{2 \sqrt{\pi}}\right) e^{i \pi / 4} \int_{-\infty}^{\infty}\left\{\mathscr{R} \sqrt{k[a+\zeta(z)]} e^{-i 2 k[a+\zeta(z) 1}-8 \sqrt{\pi} k[a+\zeta(z)] \beta_{R}^{\prime}(\infty) e^{i \Phi_{R}}\right. \\
& \times \exp \left\{-2\left(\pi-\theta_{R}\right) k[a+\zeta(z)] \beta_{R}^{\prime}(\infty)\right\} \exp \left\{i k[a+\zeta(z)]\left[\left(c / c_{R}\right)\left(2 \pi-2 \theta_{R}\right)-2 \cos \theta_{R}\right]\right\} \\
& \left.\times \sum_{m=0}^{\infty} \exp \left\{-2 \pi m k[a+\zeta(z)] \beta_{R}^{\prime}(\infty)\right\} \exp \left\{i 2 \pi m k[a+\zeta(z)] c / c_{R}\right\}\right\} \frac{e^{i k \sqrt{r^{2}+z^{2}}}}{\sqrt{r^{2}+z^{2}}} d z,
\end{aligned}
$$

where the mean radius $a$ is a constant.

As with the corresponding solutions to the prolate spheroid and uniformly bent cylinder, this integral is quite complex although, after the mean is calculated, it can either be solved exactly for all $k r$ or by the stationary phase approximation for $k r \gg 1$. In contrast to the solutions involving the other shapes, the complexity is not from a property of the cylinder that is varying along the axis but rather the fact that the cylinder is infinite in length and $r_{s}$ and the phase of the infinitesimal slices are varying along the length. The only term depending upon $z$ after the mean is taken is the quantity outside the brackets. The exact solution to the integral of this term is given in Eq. (20) of Ref. 9 and involves a zero-order Hankel function. In order to present the solution in a more intuitive manner involving the phases we solve the integral of that term in the desired farfield limit by use of the stationary phase method given in Eqs. (45) and (46). Ignoring the amplitude variation terms, taking the mean, and applying the method of stationary phase the stationary phase parameters for the above equation are $F(z)=k \sqrt{r^{2}+z^{2}}$ and $G(z)=\left(r^{2}+z^{2}\right)^{-1 / 2}$ with the $F^{\prime}=0$ stationary point at $z=0$.

Using these above terms in Eq. (46) yields the solution to the following integral:

$$
\int_{-\infty}^{\infty} \frac{e^{i k \sqrt{r^{2}+z^{2}}}}{\sqrt{r^{2}+z^{2}}} d z=\sqrt{\frac{2 \pi}{k r}} e^{i(k r+\pi / 4)},
$$

which allows us to solve for the mean of Eq. (59):

$$
\begin{aligned}
\left\langle p_{\text {scat }}\right\rangle= & P_{0} \sqrt{\frac{a}{2 r}} e^{i k r}\left\{\mathscr{R} e^{-i 2 k a} e^{-2 k^{2} \sigma^{2}}-8 \sqrt{\pi k a} \beta_{R}^{\prime}(\infty) e^{i \Phi_{R}}\right. \\
& \times \exp \left[-2\left(\pi-\theta_{R}\right) k a \beta_{R}^{\prime}(\infty)\right] \exp \left\{i k a\left[\left(c / c_{R}\right)\left(2 \pi-2 \theta_{R}\right)-2 \cos \theta_{R}\right]\right\} \sum_{m=0}^{\infty} \exp \left[-2 \pi m k a \beta_{R}^{\prime}(\infty)\right] \\
& \left.\times \exp \left(i 2 \pi m k a c / c_{R}\right) \exp \left\{-\frac{1}{2} k^{2} \sigma^{2}\left[\left(c / c_{R}\right)\left(2 \pi-2 \theta_{R}\right)-2 \cos \theta_{R}+2 \pi m c / c_{R}\right]^{2}\right\}\right]
\end{aligned}
$$

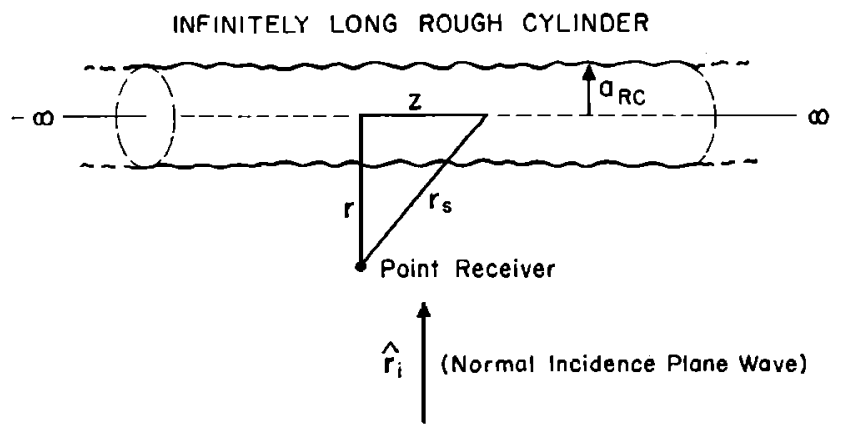

FIG. 6. Rough infinitely long cylinder along the $z$ axis. The perpendicular distance $r$ between the field (receiver) point and axis of the cylinder and distance $r_{s}$ to an arbitrary point on the axis are illustrated. which describes the mean backscattered field due to a plane wave of sound normally incident upon a randomly rough infinitely long cylinder [note that the solution in Eq. (60) is the far-field limit of the exact solution in Ref. 9].

\section{SUMMARY OF ROUGHNESS EFFECTS}

There are several interesting similarities and differences in the effects due to roughness between the $k a \ll 1$ and $k a \gg 1$ regions and among the different shapes.

For $k a \ll 1$, the roughness-induced variation of the averaged echo is independent of frequency and only depends on the roughness parameters. As a result, for small frequency, effects due to roughness may not necessarily be small. Also, 
TABLE I. Roughness-induced attenuation of mean scattered field due to various randomly rough objects: (1) planar interface, normal incidence specularly reflected wave and (2) various solid elastic elongated objects, backscattering at broadside incidence (straight finite cylinder, prolate spheroid, uniformly bent finite cylinder, and infinitely long cylinder). In the volumetric case involving tungsten carbide objects that are rough in one dimension, the $m=0$ Rayleigh surface wave is shown to dominate the specular and $m>0$ Rayleigh waves for high $k o$.

\begin{tabular}{|c|c|c|}
\hline \multirow{2}{*}{$\begin{array}{l}\text { Planar interface } \\
\text { Specular wave }\end{array}$} & \multicolumn{2}{|c|}{ Elongated volumetric objects $(k a>1)$} \\
\hline & Specular wave & Rayleigh surface elastic wave \\
\hline$e^{-2 k^{2} a^{2}}$ & $e^{-2 k^{2} a^{2}}$ & $\begin{array}{l}\exp \left(-\frac{1}{2} k^{2} \sigma^{2}\left[\left(c / c_{R}\right)\left(2 \pi-2 \theta_{R}\right)-2 \cos \theta_{R}+2 \pi m c / c_{R}\right]^{2}\right\} \\
\sim \exp \left[-2 k^{2} \sigma^{2}(0.133+1.21 m)^{2}\right] \text { (tungsten carbide) } \\
(m=0,1,2, \ldots)\end{array}$ \\
\hline
\end{tabular}

the mean scattered field increases with roughness where the opposite is true in the $k a \gg 1$ region. The lack of dependence upon frequency for the entire range of frequency is a phenomenon that is specific to volumetric scattering and should be compared with scattering by rough planar interfaces where the variations can depend strongly upon frequency. For example, Clay and Medwin show that the mean and mean-square field vary as $e^{-2 k^{2} \sigma^{2}}$ and $e^{-4 k^{2} \sigma^{2}}$, respectively, in the case of rough planar interfaces. ${ }^{50}$ The underlying reason for this difference is the fact that for $k a \ll 1$, variations in the scattering amplitude of the rough cylinder are caused by variations in the amplitude of the "local" echo from the infinitesimal slice of the cylinder that is integrated while phase-shift-induced fluctuations are negligible [Eq. (25a)]. The mathematics show that this amplitude-variation-induced fluctuation is independent of frequency. In contrast, variations in scattering due to rough planar interfaces are due, in part, to local changes in phase while the local changes in amplitude can quite often be considered negligible. The phase shifts are frequency dependent, hence causing the frequency dependence of the fluctuations for rough planar interfaces.

In a manner similar to the above discussion, we find the same types of differences between the averaged scattered field of a rough cylinder at both extremes, $k a \ll 1$ and $k a \gg 1$. As a result of the roughness-induced phase shifts of each infinitesimal ray in the the $k a \gg 1$ region (all shapes), the specular and Rayleigh surface waves are modified by the exponential term that depends upon both the rms roughness $\sigma$ and frequency. The exponential factor in the specular term (also see Table $\mathrm{I}$ ) is exactly the same as that calculated for the scattering from rough planar interfaces. ${ }^{46}$ That correspondence illustrates the fact that the front surface of the cylinder at high frequencies broadly resembles a planar interface (at least within the first Fresnel zone). Thus rough surface scattering from the front surface of the cylinder would broadly resemble scattering from a rough planar interface. As in rough planar surface scattering, the exponential factor will decrease as the product $k \sigma$ increases. That is, as the roughness becomes large when compared with the wavelength of the incident sound wave, the specular component of the mean scattered wave will correspondingly decrease exponentially. This is due to the fact that the phases of the infinitesimal rays that are summed in the integral are random for $k \sigma \gg 1$. The resultant sum of the rays is substantially decreased due to their incoherent addition. Note that for high enough frequencies, substantial decreases can occur even for small fractional roughness $\sigma / a$ (it is only the product $k \sigma$ that is important).

In a similar manner, the exponential factor in the second term (Rayleigh surface elastic wave) decreases that term as the product $k \sigma$ increases (again, see Table $\mathrm{I}$ ). This factor is different than the corresponding factor in the specular term in an interesting way. In the specular term, the decrease in the mean field was due to phase perturbations in the two-way ray that travels straight toward and away from the cylinder-hence the exponential factor that resembles the one in rough planar surface scattering. In the Rayleigh wave, the

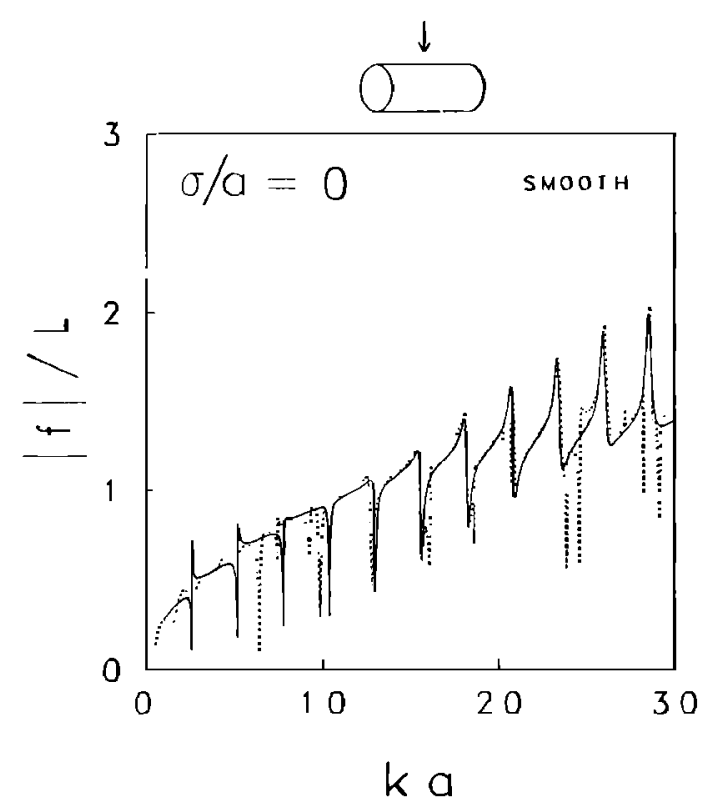

FIG. 7. Comparison between (backscattering) approximate modal-series(dashed) and SWT-based (solid) solutions for smooth straight finite cylinder at broadside incidence. The summations in both solutions are mathematically converged with the first 36 terms of their respective sums being used for the calculations. Both solutions are also approximate as they involve use of the deformed cylinder formulation. Secondly, the SWT solution uses only the "specular" and Rayleigh surface elastic wave components with the approximate expression for the coupling coefficient $G_{R}$ as derived by Marston used in the Rayleigh wave component. The cylinder is constructed of (solid) tungsten carbide with a density of $15.0 \mathrm{~g} / \mathrm{cc}$ and compressional and shear speeds of sound of 6655 and $3984 \mathrm{~m} / \mathrm{s}$, respectively. ${ }^{59}$ Using $c=1460 \mathrm{~m} / \mathrm{s}$ of the surrounding water, we obtain $h c=4.56$ and $h s=2.73$, where $h c$ and $h s$ are the respective ratios of the compressional and shear speed of sound in the cylinder material to the speed of the sound in the water. Values of $c_{R} / c=2.60, \beta_{R}(\infty)=0.00232(\operatorname{Ref} .40)$ and $\Phi_{R}=\pi / 2$ (best fit) were also used. There is reasonable comparison between the two solutions in the $15<k a<40$ region with respect to mean levels and broad oscillations (calculations up to only $k a=\mathbf{3 0}$ are shown). 
phase perturbation is due to variations in both the two-way ray and the path length of the circumnavigating wave. Thus, not only does the attenuation due to the roughness elements depend upon $k \sigma$, but also on the number of times the wave has traveled around the cylinder. As a result, the exponential factor in the Rayleigh wave term depends upon $m$. It is also interesting to note that the attenuation of the $m=0$ term is much less than that of the specular term (see Table I), hence for large $k \sigma$, the $m=0$ Rayleigh wave dominates the scattering by tungsten carbide cylinders that are rough in one dimension.

Interestingly, the scattered fields due to the objects involving all four shapes in the $k a \gg 1$ region contained the above-mentioned exponential factors. In fact, the square of the specular term for each shape is equal to the geometric scattering cross section for the smooth case (as given in Ref. 10) times the square of the exponential factor. This consistency is due to the fact that all of the objects have high aspect ratios and (1) as an approximation, the boundary conditions from the infinitely long cylinder are used in all cases resulting in the similar results and (2) the roughness was simply an additive term to the instantaneous mean cylinder radius at any point of each object. This additive term shifted the phase the same amount with each object (at least in the first Fresnel zone) hence giving the same attenuation factors. Thus the results given in this article probably provide useful insight into the scattering properties of other simple high aspect ratio objects.

When examining the mean square of the field, a dependence upon the autocorrelation function of the surface was demonstrated. This dependence was coupled with the dependences upon frequency and rms roughness. A simplified low roughness expansion of these results showed the mean square to depend upon the correlation length, rms roughness, and frequency.

Finally, in the limit of zero roughness, the expression for the rough infinitely long cylinder converges to the corresponding solution for the smooth case obtained by inserting Eq. ( 15) in Eq. (2). Thus the approximate integral formulation is self-consistent in that it was based on partitioning the scattering solution of an infinitely long cylinder into the summation of the individual contributions from all infinitesimal slices of the cylinder and the resultant sum is equal to the original solution for the infinitely long cylinder.

\section{NUMERICAL EXAMPLES}

In this section, we investigate the backscattering characteristics of randomly rough straight finite cylinders for $k a>1$ by numerical evaluation of both the SWT-based solution [ (Eq. (36)] and the (approximate) modal-series-based solution [statistical mean and mean square of Eq. (18) using Eqs. (4) and (21a) ]. The SWT solution in Eq. (36) is evaluated directly while the modal series solution is evaluated by use of Monte Carlo simulations. The cylinders simulated in these calculations are all made of tungsten carbide. Since the SWT solution involved the assumption that the amplitude of the roughness was Gaussian distributed, the roughness in the cylinders simulated in the Monte Carlo averages was constructed with Gaussian characteristics. The computa- tions not only illustrate some of the properties of the scattered field due to rough cylinders, but also the range of validity of this particular form of the SWT solution that: (1) contains contributions from only the specular and Rayleigh surface waves, (2) contains an approximate form for the coupling coefficient $G_{R}$ of the Rayleigh surface wave, and (3) is valid only when the slopes of the roughness elements are small. It should also be stressed that the modal-seriesbased solution, while mathematically converged in the following cases, is not exact as it is evaluated in the deformed cylinder formulation where its accuracy increases with decreasing slope of roughness.

Before we introduce effects due to roughness of the cylinders into the calculations, we first compare the SWT- and modal-series-based solutions for smooth (tungsten carbide) cylinders. Thus, the errors due to approximations made in the smooth SWT-based solution that are not related to roughness [approximations (1) and (2) above] can be illustrated separately from the effects due to roughness-related approximations [approximation (3) above]. Figure $7 \mathrm{com}$ pares the backscattering due to smooth tungsten carbide cylinders at broadside incidence as predicted by the SWT-based solution [Eq. (36), $\sigma=0$ ] and the modal series solution [Eqs. (4) and (18)]. The backscattering amplitude normalized by the length of the cylinder is plotted against $k a$. Because the SWT-based solution is more accurate at high $k a$, it is only plotted for values of $k a$ greater than unity while the modal series solution is plotted over a broader range of $k a$. Note that, although the value $\Phi_{R}=\pi / 4$ as predicted by Marston and Williams in Ref. 57 resulted in a reasonable fit between the SWT and modal series solution, we used the value $\Phi_{R}=\pi / 2$ as it produced the best fit. While the author is unable to explain this difference, perhaps it is related to the fact that the analysis in Ref. 57 involved numerical determination of the speed of the Rayleigh wave while the speed used in this article was approximate and constant. The exact solution shows there to be significant dispersion, especially in the $k a \leqslant 15$ region. ${ }^{39}$

The modal series solution is characterized by two regions, the Rayleigh scattering region $(k a \ll 1)$ and the geometric scattering region $(k a>1)$. In the Rayleigh region, the backscattering amplitude increases rapidly and monotonically with $k a$ while for $k a>1$ (in the geometric region), it oscillates about a mean. The SWT solution is only plotted in the $k a>1$ region as that is the region in which it is most accurate. The trend of the SWT solution is dominated by the contribution due to the specular term while the oscillations are due to interference between the specular and the Rayleigh surface elastic waves.

Figure 7 shows the SWT solution to more closely resemble the exact solution as $k a$ is increased. As illustrated in this plot and others not shown, the approximate solution appears to be reasonable in the $15<k a<40$ range. The major (broader) features of the resonances in the SWT curve resemble those in the modal series curve for $k a$ above about 15 . Below $k a=10$, only the general trend of the SWT solution follows that of the modal series solution. Dispersion of the Rayleigh wave and exclusion of the Franz wave account for some of the differences in that region. The fine detail in the 
scattering characteristics over other parts of the $k a$ range as predicted by the modal series solution is not predicted by this form of the SWT solution because, in part, of the exclusion of the Whispering Gallery waves.

Predicting the sound scattering by rough cylinders involved direct evaluation of Eq. (36) for the SWT solution and a statistical average of Monte Carlo simulations of Eqs. (4), (18), and (21a) for the modal series solution. As in Fig. 7, the calculations involved backscattering at broadside incidence. The Monte Carlo simulations first required generation of an ensemble of statistically independent randomly rough cylinders. The scattered field as described in Eqs. (4) and (18) was calculated for each roughness realization given in Eq. (21a) and the results were averaged over the ensemble at each $k a$ value.

The surface of each straight cylinder was calculated using Eq. (21a) where the random roughness parameter $\zeta(z)$ was generated by use of a sum of sinusoids of random amplitudes, wave numbers, and phases:

$$
\zeta(z)=\sigma \sqrt{6 / N} \sum_{n=1}^{N} \mu_{n} \sin \left(k_{n} z+\delta_{n}\right) .
$$

Inserting this expression into Eq. (2 Ia) gives

$$
\begin{aligned}
a_{R C}(z) & =a+\zeta(z) \\
& =a\left(1+\frac{\sigma}{a} \sqrt{\frac{6}{N}} \sum_{n=1}^{N} \mu_{n} \sin \left(k_{n} z+\delta_{n}\right)\right) .
\end{aligned}
$$

The amplitude $\mu_{n}$, wave number $k_{n}$, and phase $\delta_{n}$, were all randomized in the simulations. The summation procedure is analogous to the manner in which time series of random noise is described: the wave number is replaced by the angular frequency and the distance $z$ is replaced by time. ${ }^{60}$ The factor $\sqrt{6 / N}$ is a normalization term. The $\sqrt{6}$ reflects, in part, the fact that $\mu_{n}$ is uniformly distributed between 0 and 1. The ratio $\sigma / a$ in Eq. (63) is the fractional rms roughness. It is one of the parameters varied in the calculations of the scattered field due to the rough cylinders.

Figure 8(a) illustrates several of the cylinders generated using Eq. (63) and a histogram of the amplitudes of the cylinders. The roughness is exaggerated in the histogram so as to illustrate the fit between the histogram and Gaussian
PDF that is superimposed. The random variables, $\mu_{n}, k_{n}$, and $\delta_{n}$, were created with a random number generator in the computer. Since the values of the wavenumber extended over a finite range, the roughness is, in the analogy of time series analysis, "band-limited noise". As in time series, the resultant amplitude should follow Gaussian statistics which is demonstrated in the figure. Also shown in this figure is the autocorrelation function $\Phi$ of the rough surface of one of the cylinders. The function is shown to decrease more-or-less linearly from the origin down to the zero level beyond which the function oscillates (although a closer look on an expanded scale shows the peak to be rounded very near the origin). As discussed in Appendix B, the first zero crossing is being considered in this article as the correlation distance $\mathscr{L}$ and is shown in Fig. 8(b) to be approximately equal to 0.04 times the length of the cylinder in this case.

Scattering predictions using randomly rough cylinders as generated by the above process are illustrated in Fig. 9 . The expression for the radius given in Eq. (63) was used in the approximate modal series solution [Eqs. (4) and (18)]. The mean of the field due to $60-90$ cylinders was calculated and plotted against $k a$ as shown in the dashed curve. The total number of cylinders used varied with roughness as only 60 cylinders were required for convergence in the lowroughness case $(\sigma / a \leqslant 0.04)$ while 90 cylinders were required for greater roughnesses. The calculations were performed for tungsten carbide cylinders at broadside incidence for a variety of roughnesses. Supcrimposed upon the modalseries-based plots are calculations using the SWT approach [solid curve, Eq. (36) ]. A complete set of calculations was not made beyond $k a=30$ because of the great computation times involved in the Monte Carlo simulations (higher $k a$ values require more modal terms).

As in Fig. 7, the curves are characterized by the Rayleigh $(k a \ll 1)$ and geometric $(k a>1)$ scattering regions with additional effects due to roughness in the high $k a$ region. Beyond a certain value of $k a$, the trend of scattering amplitude will decrease. This value of $k a$ depends upon the rms roughness. As shown in Eq. (36), the specularly reflect-

(b)

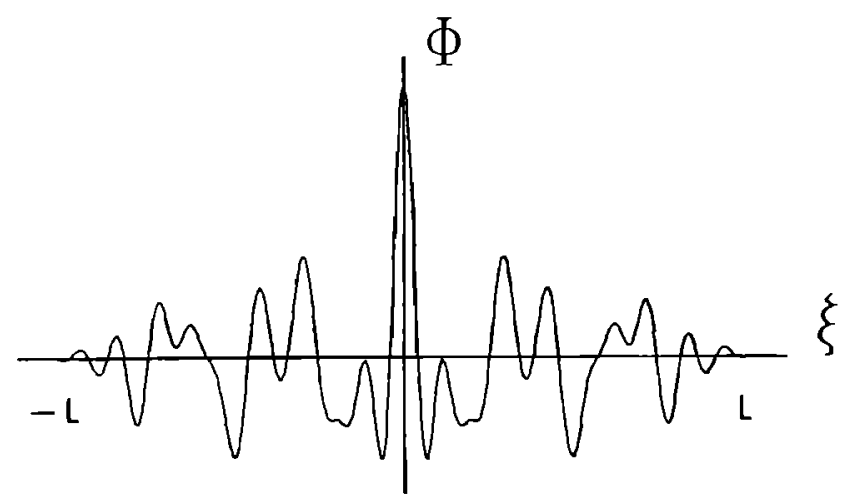

FIG. 8. (a) Ensemble of randomly rough straight finite cylinders and sample of radius statistics. The roughness component of the cylinder was generated by a summation of sinusoids whose amplitudes. phases. and wave numbers were randomized. The histogram (solid line), representing the radius statistics at a given point on a cylinder was constructed by sampling the radius at the same respective point on each (statistically independent) cylinder. Superimposed upon the histogram is a Gaussian PDF (dotted line) with the same standard deviation as in the simulated surfaces. A total of 100 sinusoids were used to generate 100 points on each of the 300 rough cylinders generated although only a subset of the data were used in the scattering simulations. The amplitudes of the sinusoids were distributed uniformly in the $0-1$ range, their wave numbers in the 0.5 to 2.5 range and the phase, 0 to $2 \pi$. The position variable covered the 0 to 50 range. (b) Autocorrelation function of the rough surface of one of the cylinders ( 60 points long). 
MEAN
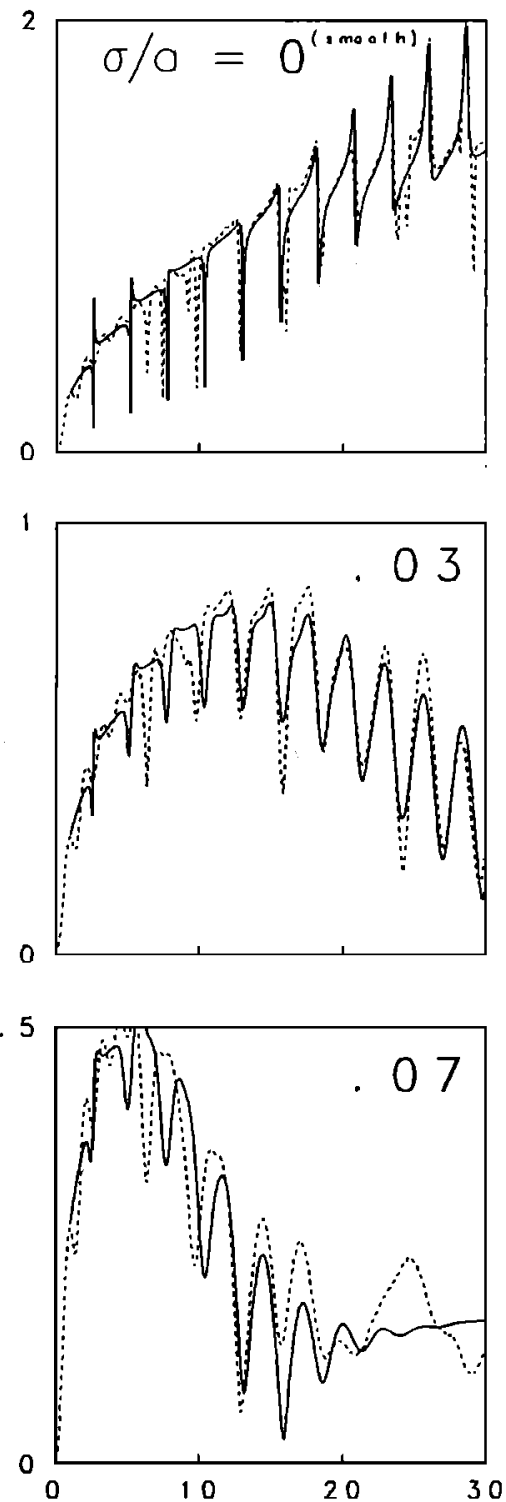
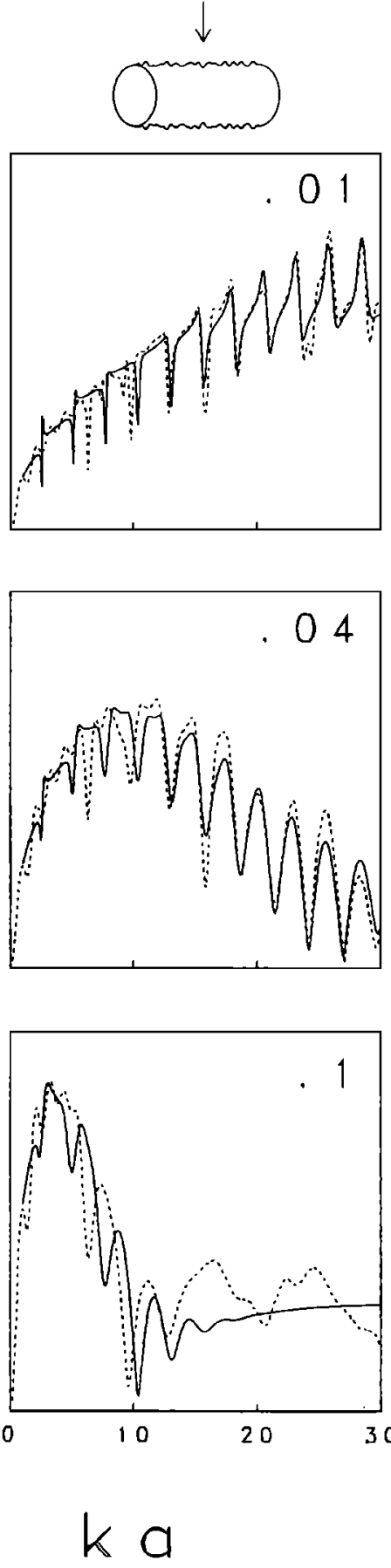

ROUGH

CYLINDERS
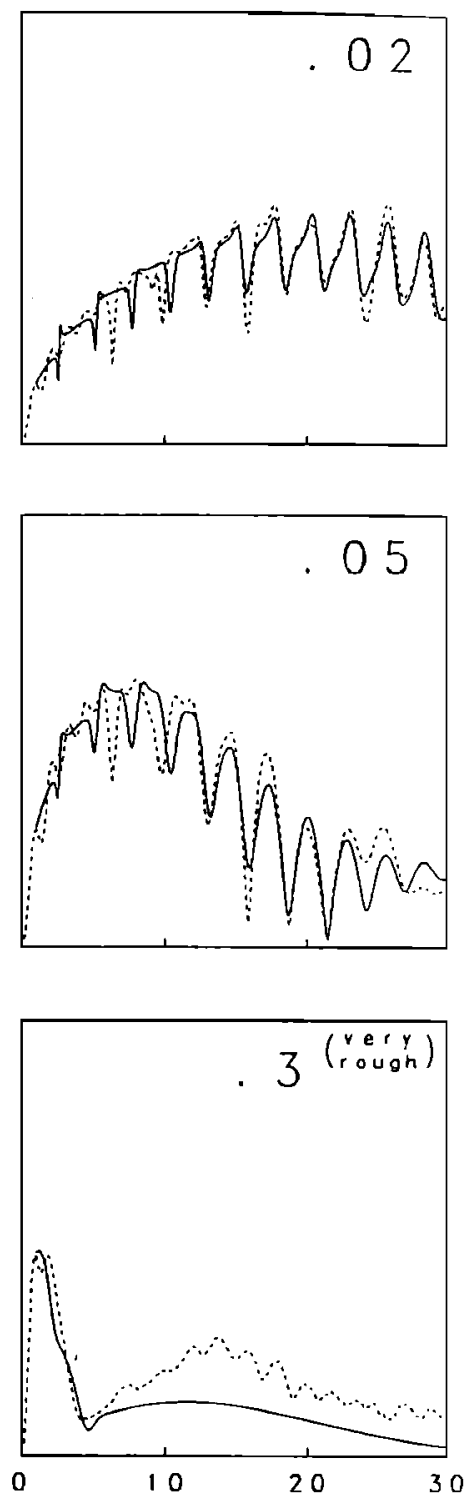

..... modal series

$-S W T$

FIG. 9. Backscattering by rough straight finite elastic cylinders of varying roughnesses ranging from perfectly smooth $(\sigma / a=0)$ to very rough $(\sigma / a=0.3)$. Solutions of the mean field were generated by Monte Carlo simulations of the approximate modal-series-based solution (dashed) and numerical evaluation of the analytical solution based on the SWT approach (solid) as derived in this article. $60-90$ points per cylinder and 60-90 cylinders were used in the Monte Carlo simulations ( 60 points, 60 cylinders for $\sigma / a \leqslant 0.04$ and $(90,90)$ for $\sigma / a>0.05$ ). The summations in both solutions for these tungsten carbide cylinders are mathematically converged. More details of the material properties of the cylinders are given in the caption to Fig. 7 . The sharp spikes in the modal series plots are shown to disappear as the cylinders are roughened. All curves are altered downward due to roughness effects for all $k \sigma \gtrsim 0.5$.

ed component of the scattering amplitude varies as $e^{-2 k^{2} \sigma^{2}}$ or $e^{-2(k a)^{2}(\sigma / a)^{2}}$. As discussed before, this component dictates the trend of the scattering for the entire range of $k a$ for smooth cylinders and, as will be shown later in the section, the low-to-moderate roughness region of rough cylinders. The point beyond which the roughness becomes important in the trend occurs approximately when the exponent becomes -0.5 at $k a=[2 \sigma / a]^{-1}$ (or equivalently, $k \sigma=0.5$ ). This estimate of the "turning point" of the trend is consistent with the curves in Fig. 9. For example, for a fractional roughness of $\sigma / a=0.05$, the turning point would be at $k a=1 /[2(0.05)]=10$, which is where the turning point occurs on the corresponding plot. This simple formula predicts the turning point for the curves at other roughnesses as well. As discussed in Section $I$, the expression $e^{-2 k^{2} \sigma^{2}}$ appeared originally in the description of the scattering by 


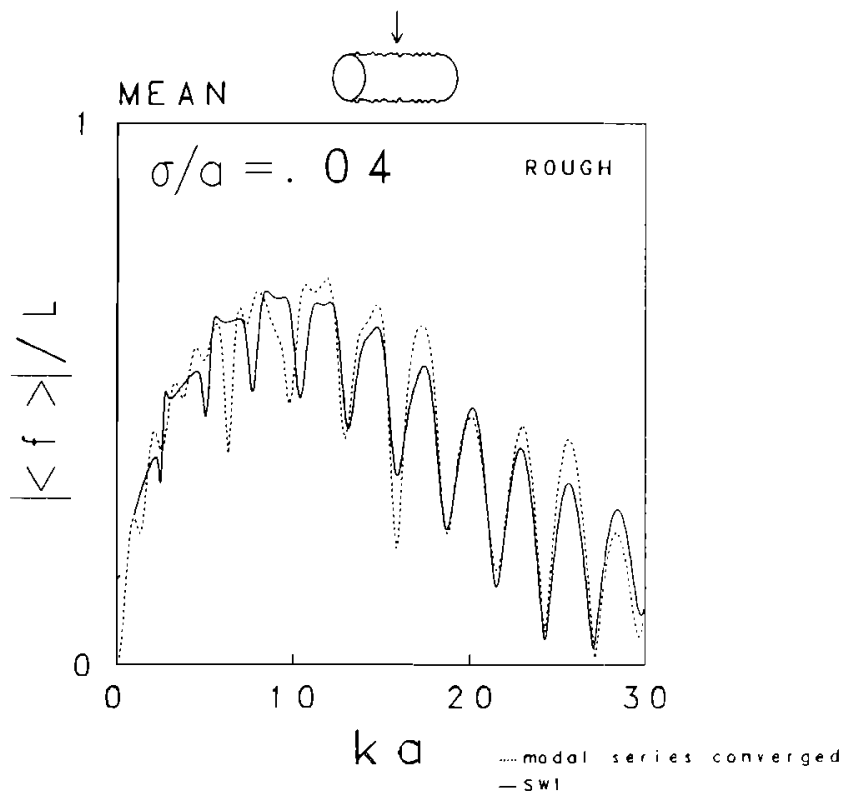

FIG. 10. Enlarged plot of the $\sigma / a=0.04$ case in Fig. 9. The correlation at high $k a$ values between the resonance structures predicted by the two independent techniques is illustrated.

rough planar interfaces. ${ }^{46}$ The fact that both curves, which were based on the modal series and SWT solutions, are consistent with that expression demonstrates the similarity between the scattering by the two types of boundaries (volumetric and planar).

For roughnesses below $\sigma / a=0.05$ and $k a \gtrsim 15$, there is reasonable similarity between the modal series solution and SWT solution demonstrating at least a range of validity of the analytical results. The curves are similar both in trend and occurrence of oscillations (above $k a=15$ ) about the trend. The amplitudes, in general, do not exactly coincide. As in the zero roughness case, some of the differences are due, at least in part, to the fact that the SWT approach included only the specular and Rayleigh surface waves (ignoring surface waves such as the Whispering Gallery waves) and the Rayleigh wave coupling coefficient was used in an approximate form. It is interesting to observe that once the cylinder is rough, some of the fine features disappear resulting in a better fit between the theory and simulations. Also, both theory and simulations predict a "rounding" of the curves once roughness is introduced. At fractional roughnesses greater than or comparable to 0.05 , the two curves depart at high $k a$. This departure is not surprising as in this high fractional roughness region, the slopes of the roughness are not small introducing the possibility for error both in the simulations and theory. Note that although the simulations and theory are based on the same small-slope assumptions, they may break down in different ways.

Figure 10 is a blowup of the 0.04 fractional roughness case shown in smaller form in Fig. 9. In this enlarged version, the correlation between the SWT and modal series solutions is evident. As discussed above, both the trend for $k a>1$ and oscillations about the trend for $k a>15$ are similar between the two curves, while the amplitudes, in general, are different.
Figure 11 illustrates the root-mean square of the backscattering amplitude of the cylinders modeled in Fig. 9. Only the Monte Carlo simulations of the modal-series-based solution are given, but clearly, the curves are behaving in a manner similar to the means in Fig. 9. In particular, the curves resemble the zero roughness case in Fig. 7 (dashed) up to a value of $k a$ corresponding to $k \sigma \sim 0.5$ beyond which point the curves deviate in a downward direction. As in the case of the mean scattering amplitude, the deviation represents attenuation of the signal due to the interferences caused by the roughness.

In order to better illustrate the loss of the various waves due to roughness, the mean scattering amplitude is plotted up to a value of $k a=100$ in Fig. 12 for two roughnesses. Only the SWT solution could be plotted as the modal series representation involved too much computer time in the high $k a$ region. The oscillatory nature of the scattering due to interference between the Rayleigh and specular waves is shown to decay in the high $k a$ region. This is due to the fact that for high $k a$, the specular (also shown separately) and higher order $(m>0)$ Rayleigh waves are attenuated at a much higher rate due to the (one-dimensional) roughness than the $m=0$ Rayleigh wave. The effects are also shown mathematically in Table I where expressions for the roughness-induced attenuations are given for each wave.

The $m=0$ Rayleigh wave dominates the specular wave for this tungsten carbide material because of offsetting effects illustrated in the exponent in the third column in Table I. The phase shift induced at the boundary due to the roughness, which is represented by the $-2 \cos \theta_{R}$ term, is opposite in sign to the shift induced by the change in travel path along the boundary which is represented by the $\left(c / c_{R}\right)\left(2 \pi-2 \theta_{R}\right)$ term. With the ratio of $c_{R} / c$ being 2.6 , these two terms are comparable in magnitude resulting in a small difference equal to 0.133 .

All of the simulations to this point plot some form of the scattering amplitude against $k a$. As a result, the resonance structure plays a major role in the pattern of the plots. It is also useful to plot the scattering amplitude in such a way so that the structure is held constant while the major trends are illustrated. That can be done by holding $k a$ fixed and varying $\sigma / a$. Figure 13 illustrates the mean and root mean square of the backscattering amplitude due to rough straight finite cylinders (tungsten carbide as before) with $k a$ fixed at 30 and $\sigma / a$ ranging from 0 to 0.05 . Also plotted is the Eckart attenuation factor $e^{-2 k^{2} \sigma^{2}}$ as the equations for the mean [Eq. (36)] and mean square [Eq. (43)] both contain that term in the specular component of the field. For the purposes of plotting, the factor is scaled by the scattering amplitude for the zero roughness case.

There is remarkable agreement between the simple plot of the Eckart attenuation factor and the simulations for the range $0<\sigma / a<0.02$ which corresponds to low to moderate roughness $0<k \sigma<0.6$. All three plots essentially coincide in this range demonstrating the dominance of the Eckart factor in the scattering process. The range $\sigma / a>0.03$ corresponds to the high roughness region $k \sigma>1$ and there are deviations between the three plots. Deviations from the simple predictions are expected in the high roughness region because of 


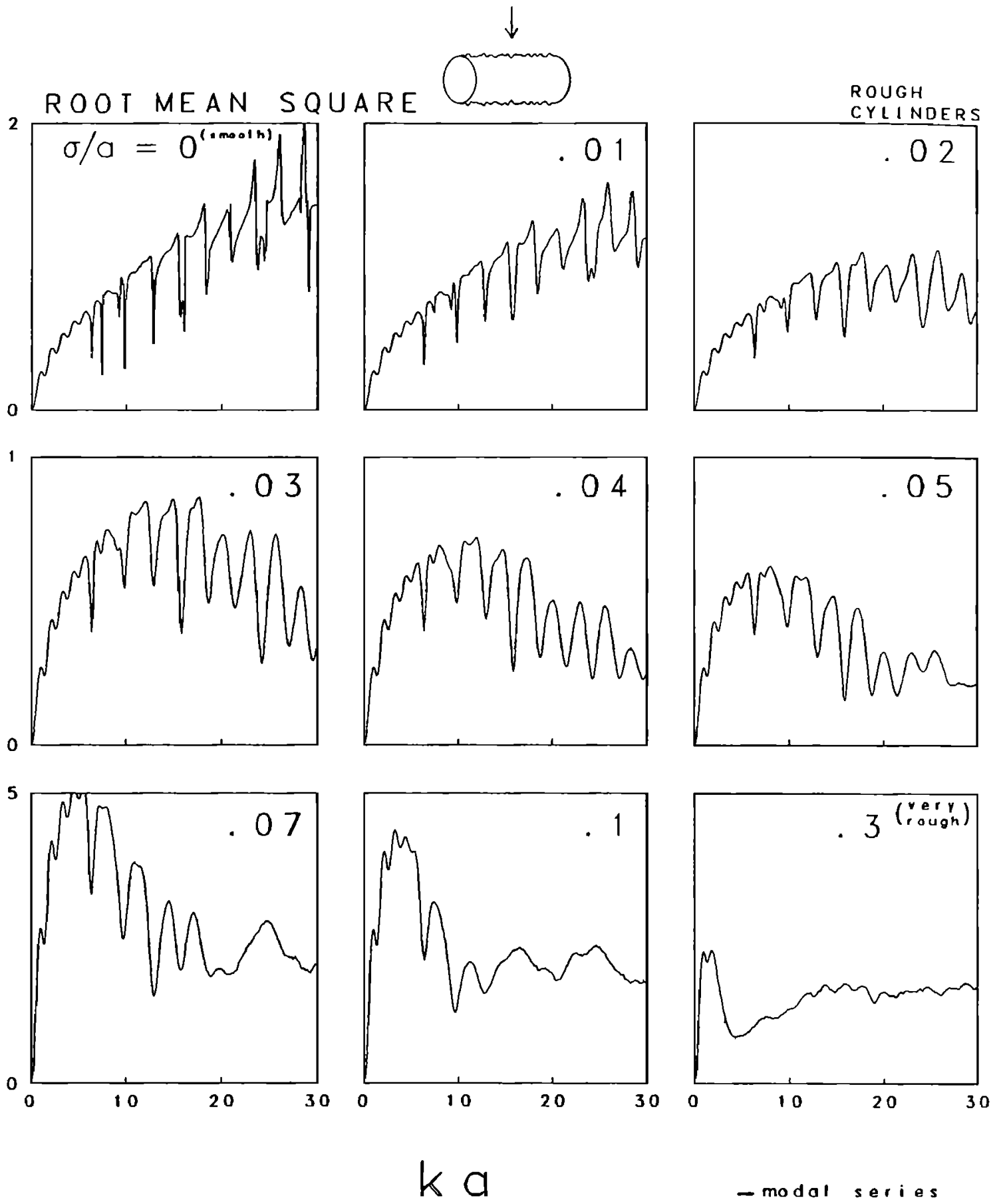

FIG. 11. Root-mean-square backscattering amplitude due to rough straignt finite elastic cylinders used in Fig. 9. Only the Monte Carlo simulations of the approximate modal-series-based solution were calculated (SWT solution not shown).

the dominance of the $m=0$ Rayleigh wave (see Fig. 12).

\section{CONCLUSIONS}

By randomizing the radius in the deformed cylinder formulation, we have obtained approximate analytical expressions describing the backscattering of sound by the following rough elongated elastic objects: straight finite length cylinder, prolate spheroid (high aspect ratio), uniformly bent finite cylinder, and infinitely long cylinder. Depending on the value of $k a$ either the modal-series-based $(k a \leqslant 1)$ or SWT-based $(k a \gg 1)$ solutions were used. Both the mean and mean-square scattered fields for both $k a \leqslant 1$ and $k a \gg 1$ were formulated for the straight finite cylinder while only the mean field for $k a \gg 1$ was formulated for the other shapes. Comparisons of the $k a \gg 1$ expressions involving the straight finite cylinders with numerical simulations verify the validity of applying the SWT approach and retaining only the specular and Rayleigh waves for the given range of conditions.

The results of this analysis at $k a \gg 1$ are quite intuitive in that one would expect the various waves to become attenuated due to roughness effects. One should also have expected the Rayleigh wave to become increasingly attenuated (in addition to radiation damping) as the number of times it has 

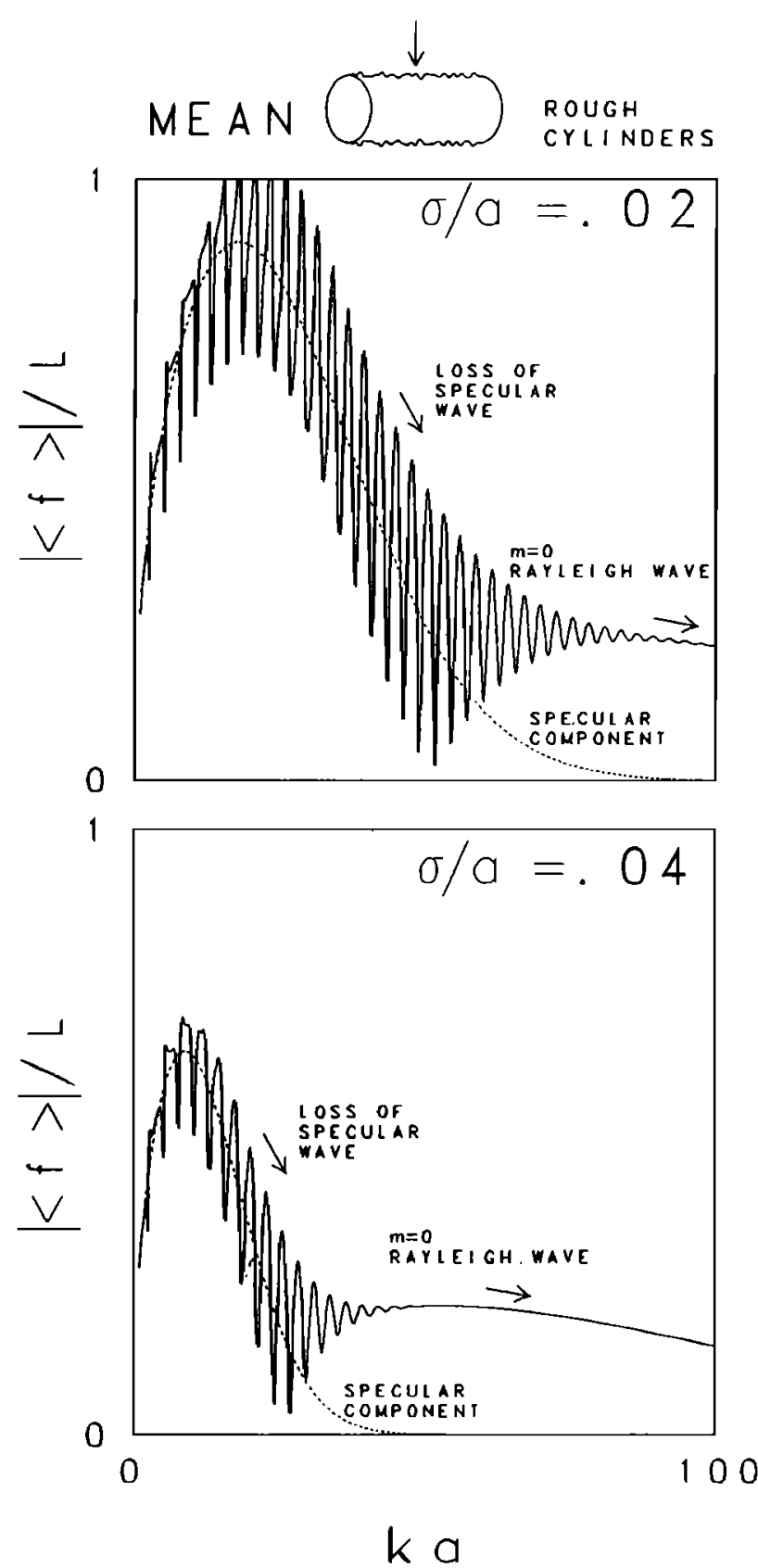

FIG. 12. Illustration of dominance of first ( $m=0$ ) Rayleigh surface elastic wave over the specular and $m>0$ Rayleigh waves due to roughness at high $k a$. While all waves are attenuated by the (one-dimensional) roughness, the $m=0$ Rayleigh wave is attenuated the least ( $2 \%$ and $4 \%$ roughness cases shown). More details of the material properties of the tungsten carbide cylinders are given in the caption to Fig. 7.— SWT (specular plus Rayleigh waves); - . SWT (specular component only).

circumnavigated the object increases. While this research only involved dense solid elastic materials in a region where the transmitted bulk waves can be assumed to be negligible, it is also intuitive that under different conditions where the waves are important such as with a higher $k a$ or less dense materials, the reradiated transmitted bulk waves will behave in a similar manner. One might guess that the attenuation would depend upon both $k \sigma$ and on the number of times it reflected internally within the object before it is reradiated. The same arguments apply to Franz and Whispering Gallery waves as well as those waves involved with shelled objects.

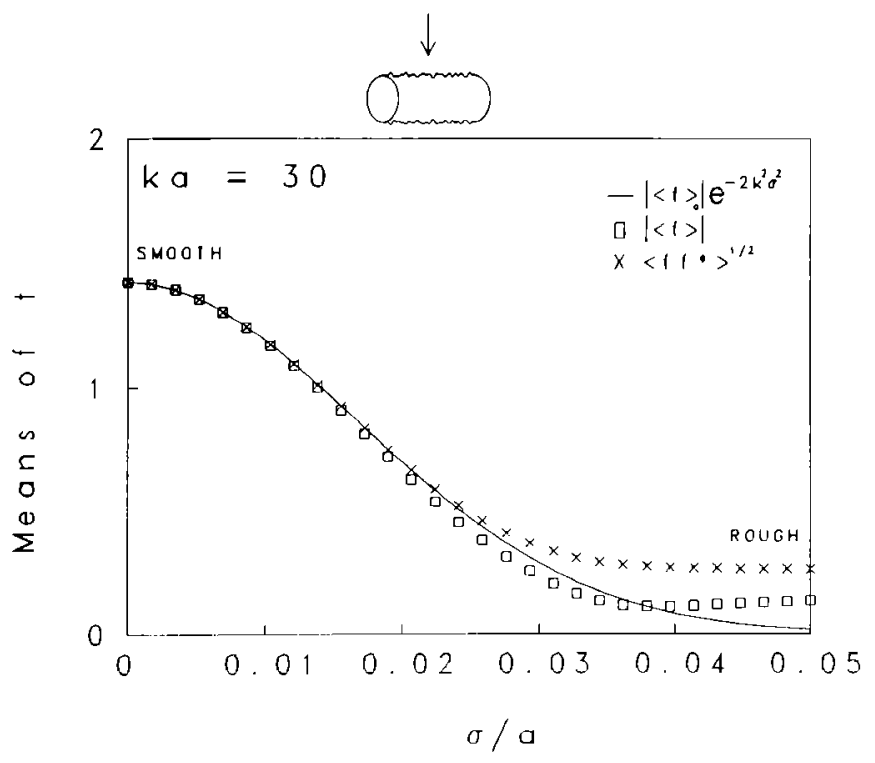

FIG. 13. Mean, root-mean-square, and Eckart attenuation factor $e^{-2 k^{2} \sigma^{2}}$ for $k a=30$ over a range of values of $\sigma / a$. For the purpose of illustration, the Eckart factor is scaled by the zero roughness scattering amplitude. The difference between the points and curve is due in part to the dominance of the $m=0$ Rayleigh wave at high $k \sigma$. More details of the material properties of the tungsten carbide cylinders are given in the caption of Fig. 7.

This solution describing the scattering by randomly rough elongated elastic objects helps provide much insight into the previous papers where the scattering of sound by zooplankton was modeled. In those papers, an optimum fit between the model and data required modification of the modal series solution. The modification, in the form of truncation of the series, was based on intuition, not derivation. The results in this paper provide some of the quantitative foundation necessary in the scattering description of marine organisms. However, much more needs to be done. Depending on the type of organism, the material of the bodies ranges from weakly scattering fluid to fluid-like-filled shells to gaseous. Thus it will be important to model the scattering properties of a broader range of boundary conditions than that presented here.

\section{ACKNOWLEDGMENTS}

The author is grateful to the following people for their advice on this complex problem: Philip L. Marston of the Department of Physics, Washington State University, Pullman Washington, Michael F. Werby of the Naval Ocean and Atmospheric Research Laboratory, Bay St. Louis, Missouri, Guillermo C. Gaunaurd of the Naval Surface Weapons Center, White Oak Lab, Silver Spring Maryland, I-Tai Lu of the Department of Electrical Engineering and Computer Sciences, Weber Research Institute, Polytechnic University, Farmingdale, New York, Steven G. Kargl of the Naval Coastal Systems Center, Panama City, Florida, and Clarence S. Clay of the Department of Geology and Geophysics, University of Wisconsin, Madison, Wisconsin. The author is also indebted to the following people at the Woods Hole Oceanographic Institution, Woods Hole, Massachusetts: Bob Eastwood for his aid in the numerical simulations on the computer, Dezhang Chu for checking certain critical simulations and equations, Shirley Bowman for preparing the 
manuscript for this article, and Betsy Pratt for drafting some of the figures. This work was supported by the U.S. Office of Naval Research (Grant No. N00014-89-J-1729). This is Woods Hole Oceanographic Institution contribution No. 7496.

\section{APPENDIX A: GAUSSIAN PDFS AND RELATED STATISTICAL AVERAGES}

When calculating the statistical mean and mean-square scattered field, it is mathematically convenient and physically reasonable to assume that the distribution of the roughness component $\zeta$ is the Gaussian PDF $W:^{56}$

$$
W(\zeta)=(1 / \sigma \sqrt{2 \pi}) e^{-\zeta^{2} / 2 \sigma^{2}} .
$$

This PDF is useful in calculations of the mean field where there is only one stochastic variable $\zeta$. However, when the mean square is calculated, there will be cross terms involving two stochastic variables, $\zeta^{\prime}$ and $\zeta^{\prime \prime}$, whose values are interdependent and the bivariate Gaussian PDF is then required: $^{.6}$

$$
\begin{aligned}
\mathscr{W}_{1,2}= & \left(2 \pi \sigma^{2}\left[1-\Phi^{2}(\xi)\right]^{1 / 2}\right)^{-1} \exp \left\{-\left[\xi^{\prime 2}+\zeta^{\prime 2}\right.\right. \\
& \left.\left.-2 \zeta^{\prime} \zeta^{\prime \prime} \Phi(\xi)\right] / 2\left[1-\Phi^{2}(\xi)\right] \sigma^{2}\right\} . \quad \text { (A2) }
\end{aligned}
$$

Some averages involving both distributions are

$$
\begin{aligned}
& \langle 1\rangle=1, \\
& \left\langle\zeta^{\prime}\right\rangle=0, \\
& \left\langle\zeta^{\prime 2}\right\rangle=\sigma^{2},
\end{aligned}
$$

while other averages involving only the bivariate distribution are

$$
\begin{aligned}
& \left\langle\zeta^{\prime} \zeta^{\prime \prime}\right\rangle=\sigma^{2} \Phi(\xi) \\
& \left\langle\zeta^{\prime} \zeta^{\prime \prime 2}\right\rangle=0 \\
& \left\langle\zeta^{\prime 2} \xi^{\prime \prime 2}\right\rangle=\sigma^{4}\left[1+2 \Phi^{2}(\xi)\right]
\end{aligned}
$$

\section{APPENDIX B: EVALUATING CERTAIN INTEGRALS OF THE FORM $\iint F[\Phi(\xi)] d z d z^{\prime \prime}$}

When calculating the mean square of the scattered field due to rough cylinders of length $L$, the following type of integral is encountered:

$$
I=\int_{-L / 2}^{L / 2} \int_{-L / 2}^{L / 2} F[\Phi(\xi)] d z^{\prime} d z^{\prime \prime},
$$

where, for the moment, $F$ is an arbitrary function of the autocorrelation function of the rough surface. In order to facilitate evaluation, we apply a transformation of variables quite often used in evaluation of the Helmholtz-Kirchhoff integral:

$$
\xi=z^{\prime \prime}-z^{\prime}, \quad z=z^{\prime},
$$

hence

$$
\begin{aligned}
& z^{\prime}=z, \quad z^{\prime \prime}=z+\xi, \\
& d z^{\prime} d z^{\prime \prime}=d z d \xi \quad(\text { Jacobian =1). }
\end{aligned}
$$

[See Fig. B1 for illustration of this transformation to the present problem of $-L / 2<\left(z^{\prime}, z^{\prime \prime}\right)<L / 2$.]

The choice of the transformation $\xi=z^{\prime \prime}-z^{\prime}$ is quite obvious as the autocorrelation function $\Phi(\xi)$ is an explicit

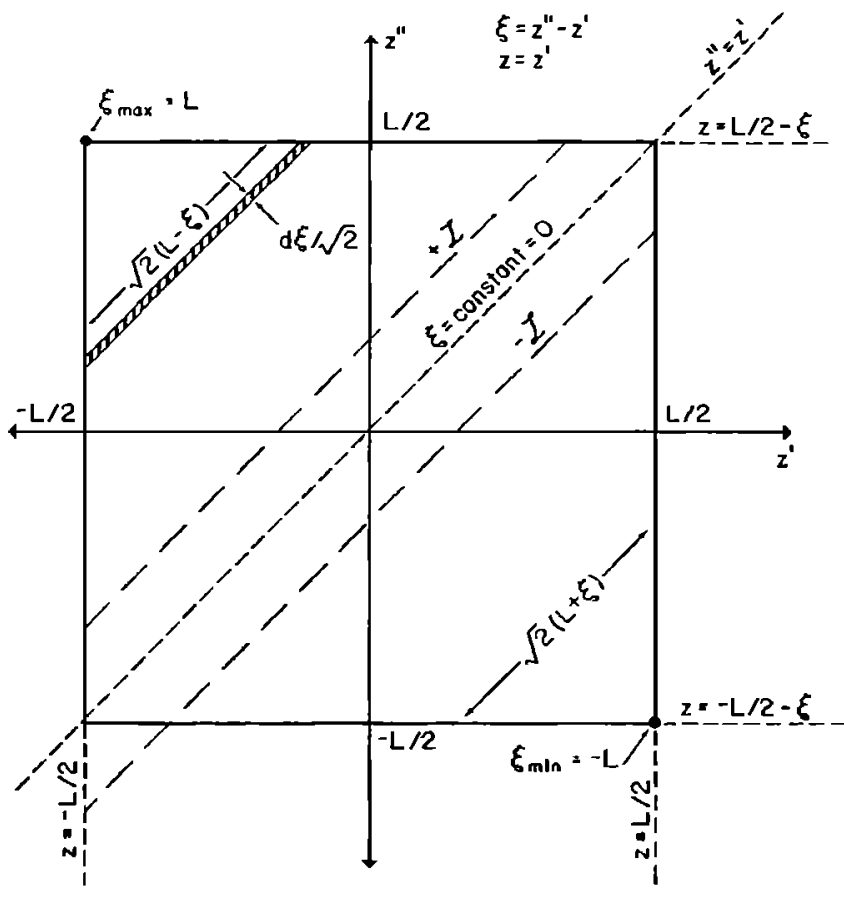

FIG. Bl. Illustration of transformation of variables $\left(\xi=z^{n}-z^{\prime}, z=z^{\prime}\right)$ and information relevant to integration along axis of straight finite cylinder of length $L$.

function of $\xi$. The choice of the transformation $z=z^{\prime}$ is somewhat arbitrary and, as stated in Skudrzyk, the new variables need not be mutually orthogonal. ${ }^{56}$ One could also have chosen, for example, $z=z^{\prime \prime}+z^{\prime}$. The Jacobian then, for the resultant relations $z^{\prime}=z / 2-\xi / 2$ and $z^{\prime \prime}=z / 2+\xi / 2$, would be $\frac{1}{2}$. Applying this alternate transformation to $\mathrm{Eq}$. (B1) would have, through the integration technique that is about to be discussed, resulted exactly in Eq. (B5) which is the equation based on the transformation given in Eqs. (B2a) and (B2b).

Observing that the integrand in the case of the straight finite cylinder depends only upon $\xi$, we first integrate along a path of constant $\xi$. Using an intuitive method described in Skudrzyk $^{36}$ and illustrated in Fig. B1 of this article, the integration can be divided into narrow strips of constant $\xi$. The area of each strip (see shaded portion in Fig. B1) is determined by multiplying its width $d \xi / \sqrt{2}$ by its length where the length is given by

length of integration strip

$$
\begin{aligned}
& =\sqrt{2} \int_{-L / 2}^{L / 2-\xi} d z=\sqrt{2}(L-\xi), \quad \xi \geqslant 0, \\
& =\sqrt{2} \int_{-L / 2-\xi}^{L / 2} d z=\sqrt{2}(L+\xi), \quad \xi \leqslant 0 .
\end{aligned}
$$

The $2^{-1 / 2}$ factor to the differential $d \xi$ was derived simply by taking the ratio of geometric length $\sqrt{2} L$ of the diagonal that is perpendicular to the strip to the full range $2 L$ spanned by $\xi$. Both $\int d z$ integrations without the $\sqrt{2}$ factor represent the projected lengths along the $z$ axis [the limits of the integrals were derived from the transformation given in Eq. (B2a) ]. The product $(\sqrt{2} d z) \times(d \xi / \sqrt{2})=d z d \xi$ pre- 
serves the unity value of the Jacobian given in Eq. (B2c) (note that Skudrzyk failed to include the factors of $\sqrt{2}$ and $2^{-1 / 2}$ but since the factors were offsetting, he obtained the correct result.). The lengths in Eq. (B3) range from 0 at the corners where $\xi$ reaches the maximum $(L)$ and minimum $(-L)$ values to $\sqrt{2} L$ along the diagonal where $\xi=0$.

Applying the transformation described in Eqs. (B2a)(B2c) to Eq. (B1) and integrating along the length of the strip gives

$$
\begin{aligned}
I= & \int_{-L}^{0}\left(\sqrt{2} \int_{-L / 2-\xi}^{L / 2} d z\right) F[\Phi(\xi)] d \xi(\sqrt{2})^{-1} \\
& +\int_{0}^{L}\left(\sqrt{2} \int_{-L / 2}^{L / 2-\xi} d z\right) F[\Phi(\xi)] d \xi(\sqrt{2})^{-1} . \\
= & \int_{-L}^{0} F[\Phi(\xi)](L+\xi) d \xi+\int_{0}^{L} F[\Phi(\xi)](L-\xi) d \xi,
\end{aligned}
$$

where the lengths given in Eqs. (B3a) and (B3b) appear in the right and left terms, respectively, of Eq. (B4a). Note that Eq. (B4a) was derived by first applying the transformation, then inserting the offsetting factors of $\sqrt{2}$ and $2^{-1 / 2}$ to produce the desired differential lengths. Since autocorrelation functions are inherently even $[\Phi(-\xi)=\Phi(\xi)]$ (Ref. 56), Eq. (B4b) simplifies to

$$
I=2 \int_{0}^{L} F[\Phi(\xi)](L-\xi) d \xi .
$$

This simplification corresponds to taking advantage of the symmetry of integration about the $\xi=0$ line in Fig. B1. The total area of the square in Fig. B1 is equal to twice the area in the upper left hand region where $\xi>0$.

In order to evaluate the integral, the correlation function must be known. For reasons involving physical considerations as well as mathematical convenience, we choose the following function written in terms of a correlation distance $\mathscr{L}$ for the integrals where sign of the correlation function is not a factor:

$$
\begin{aligned}
\Phi(\xi) & =1-|\xi| / \mathscr{L}, \quad|\xi| / \mathscr{L} \leqslant 1 \\
& =0, \quad|\xi| / \mathscr{L} \geqslant 1,
\end{aligned}
$$

where, as illustrated in Fig. B2, the function decreases linearly to 0 in the $-\mathscr{L} \leqslant \xi \leqslant \mathscr{L}$ range and is equal to 0 outside that range.

Since the function given in Eq. (B6) takes on two functional forms depending on the value of $\xi$, the integral in Eq. (B5) must be partitioned into $0 \leqslant \xi \leqslant \mathscr{L}$ and $\mathscr{L} \leqslant \xi \leqslant L$ regions:

$$
I=2 \int_{0}^{\mathscr{L}} F\left[1-\frac{\xi}{\mathscr{L}}\right](L-\xi) d \xi+2 \int_{\mathscr{L}}^{L} F[0](L-\xi) d \xi .
$$

The location of the $\xi=\mathscr{L}$ dividing line is illustrated in Fig. $\mathbf{B} 1$ at an arbitrarily placed location (the $\xi=-\mathscr{L}$ line in the region of symmetry is also shown). The value of $\mathscr{L}$ ranges from less than $L$ to much less than $L$ (if it were greater than $L$, the object would not be rough).

Further evaluation of the above integral requires knowledge of the function $F$. In the main text, $F$ is equal to the

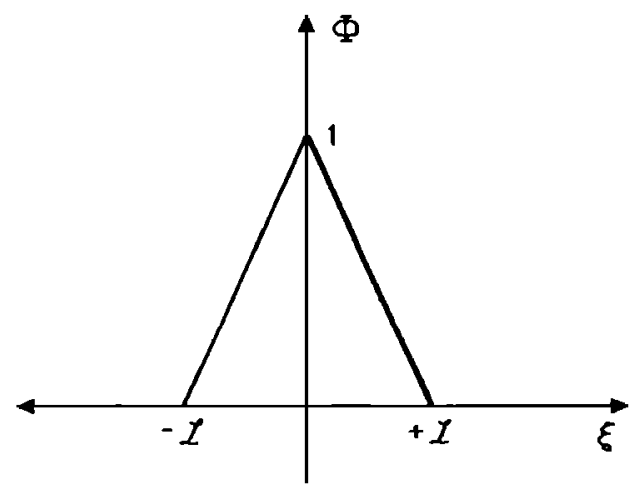

FIG. B2. One-dimensional correlation function of rough cylinder in simple convenient form. The correlation parameter $\xi$ varies along the lengthwise axis of the cylinder. This function is used in calculations where the sign of the function does not matter (such as where terms like $\Phi^{2}$ appear).

following functions: $\Phi(\xi), \Phi^{2}(\xi)$, and $e^{-4 k^{2} \sigma^{2} \alpha \Phi(\xi)}$, where $\alpha$ is a real number. One can integrate the first one directly without use of Eq. (B7) for functions more realistic than Eq. (B6):

$$
\int_{-L / 2}^{L / 2} \int_{-L / 2}^{L / 2} \Phi(\xi) d z^{\prime} d z^{\prime \prime}=0
$$

For the second expression that always remains positive regardless of choice of correlation function, we apply Eq. (B7) to Eq. (B1) to obtain

$$
\begin{aligned}
\int_{-L / 2}^{L / 2} \int_{-L / 2}^{L / 2} \Phi^{2}(\xi) d z^{\prime} d z^{\prime \prime} & =\frac{2}{3} \mathscr{L} L-\frac{\mathscr{L}^{2}}{6} \\
& \simeq \frac{2}{3} \mathscr{L} L \quad(\mathscr{L} / L \ll 1),
\end{aligned}
$$

where both the general and limiting expressions are given for the solution in Eqs. (B9) and (B10).

In this article, we will not pursue integration of the third type of expression.

Finally it is worthwhile mentioning the fact that Eq. (B1) could also have been evaluated by integrating $\xi$ first before $z$. The transformed integral is then

$$
I=\int_{-L / 2}^{L / 2} \int_{-L / 2}^{L / 2-z} F[\Phi(\xi)] d \xi d z .
$$

The solution will give the same results as in the above analysis where $z$ is integrated first, but is extremely tedious to calculate. For example, the integral in the alternate approach corresponding to Eq. (B7) involves seven double integrals (the integrals, which divide the area shown in Fig. B1 into 7 nonoverlapping regions, are partitioned that way because $\Phi(\xi)$ changes functional form at $|\xi|=\mathscr{L}$ ). The solution described in this appendix where $z$ is integrated first is the more attractive approach as it can naturally be adapted to the piecewise continuous $\Phi(\xi)$.

\section{APPENDIX C: DETERMINATION OF DOMINANT TERMS IN MEANS OF SCATTERING AMPLITUDE}

Equation (30) contains several factors in the integrand that involve the random component of the radius $\zeta(z)$. In order to make the integration of this equation convenient so 
that the dominant physical mechanisms are apparent, we investigate which terms are dominant so that the others can be ignored in the integration. There are terms whose variations due to changes in $\zeta$ are real while the remainder involve complex variations. We will show that the ones involving complex variations are the dominant terms. In particular, the dominant terms have an imaginary exponent that contains $\zeta$.

For convenience of illustration, we are evaluating individual terms of Eq. (30) in the low roughness limit where both $k \sigma \ll 1$ and $\zeta / a \ll 1$. As discussed at the end of this Appendix, the results also hold true for finite values of $k \sigma$ although $\sigma / a$ must be held well below unity so that the object does not break. Secondly, the results of this analysis involving individual terms also hold true for the actual products involving more complicated expressions containing cross products. The terms under investigation can be expanded in the $k \sigma, \zeta / a \ll 1$ limits as

real:

$$
\begin{aligned}
\text { term } 1 & \equiv \sqrt{k(a+\zeta)} \simeq \sqrt{k a}\left(1+\frac{1}{2}(\zeta / a)-\frac{1}{8}(\zeta / a)^{2}\right), \\
\text { term } 2 & \equiv k(a+\zeta) \\
& =k a(1+\zeta / a) \text { (no approximation necessary), }
\end{aligned}
$$

term $3 \equiv e^{-2\left(\pi-\theta_{R}\right) k t \beta_{R}^{\prime}(\infty)}$

$$
\begin{aligned}
\simeq 1 & -2\left(\pi-\theta_{R}\right) k a(\zeta / a) \beta_{R}^{\prime}(\infty)+2\left(\pi-\theta_{R}\right)^{2} \\
& \times(k a)^{2}(\zeta / a)^{2}\left[\beta_{R}^{\prime}(\infty)\right]^{2},
\end{aligned}
$$

term $4 \equiv e^{-2 \pi m k \zeta \beta \beta_{R}^{\prime}(\infty)}$

$$
\begin{aligned}
\simeq 1 & -2 \pi m k a(\zeta / a) \beta_{R}^{\prime}(\infty)+2 \pi^{2} m^{2}(k a)^{2} \\
& \times(\zeta / a)^{2}\left[\beta_{R}^{\prime}(\infty)\right]^{2}
\end{aligned}
$$

complex:

$$
\text { term } 5 \equiv e^{-i 2 k \zeta} \simeq 1-i 2 k a(\zeta / a)-2(k a)^{2}(\zeta / a)^{2},
$$

term $6 \equiv e^{i k \zeta\left(C_{R}+2 \pi m c / c_{R}\right)}$

$$
\begin{aligned}
\simeq 1 & +i k a(\zeta / a)\left(C_{R}+2 \pi m c / c_{R}\right) \\
& -\frac{1}{2}(k a)^{2}(\zeta / a)^{2}\left(C_{R}+2 \pi m c / c_{R}\right)^{2},
\end{aligned}
$$

where

$$
C_{R} \equiv\left(c / c_{R}\right)\left(2 \pi-2 \theta_{R}\right)-2 \cos \theta_{R},
$$

where the terms were divided in two groups: one whose variations due to changes in $\zeta$ cause real changes in the term and the other whose variations are complex.

\section{Mean}

Using the Gaussian PDF given in Eq. (A1) of Appen$\operatorname{dix} A$, the mean values of the above terms are

$$
\begin{aligned}
& \langle\operatorname{term} 1\rangle \simeq \sqrt{k a}\left(1-\frac{1}{8}(\sigma / a)^{2}\right), \\
& \langle\operatorname{term} 2\rangle=k a, \\
& \langle\operatorname{term} 3\rangle \simeq 1+2\left(\pi-\theta_{R}\right)^{2}(k a)^{2}(\sigma / a)^{2}\left[\beta_{R}^{\prime}(\infty)\right]^{2},
\end{aligned}
$$

$$
\begin{aligned}
& \langle\operatorname{term} 4\rangle \simeq 1+2 \pi^{2} m^{2}(k a)^{2}(\sigma / a)^{2}\left[\beta_{R}^{\prime}(\infty)\right]^{2},(\mathrm{C} 10) \\
& \langle\operatorname{term} 5\rangle \simeq 1-2(k a)^{2}(\sigma / a)^{2}, \\
& \langle\operatorname{term} 6\rangle \simeq 1-\frac{1}{2}(k a)^{2}(\sigma / a)^{2}\left(C_{R}+2 \pi m c / c_{R}\right)^{2},
\end{aligned}
$$

where Eqs. (A3)-(A5) from Appendix A were used. The fractional variations induced by roughness in the means above are approximately $\frac{1}{8}(\sigma / a)^{2}, 0,2\left(\pi-\theta_{R}\right)^{2}(k a)^{2}$ $\times(\sigma / a)^{2}\left[\beta_{R}^{\prime}(\infty)\right]^{2}, 2 \pi^{2} m^{2}(k a)^{2}(\sigma / a)^{2}\left[\beta_{R}^{\prime}(\infty)\right]^{2}$, $2(k a)^{2}(\sigma / a)^{2}$, and $\frac{1}{2}(k a)^{2}(\sigma / a)^{2}\left(C_{R}+2 \pi m c / c_{R}\right)^{2}$ for terms 1-6, respectively. Obviously, since the mean of term 2 does not vary with respect to roughness, then it will not influence the functional dependence of the mean. For low roughness $(k \sigma, \sigma / a \ll 1)$ but high $k a(k a \gg 1)$, a typical value of $\beta_{R}^{\prime}(\infty)=0.00232$, and moderate values of $m$ (moderate so that the expansion is valid), terms 5 and 6 that vary as $(k a)^{2}(\sigma / a)^{2}$ and $m^{2}(k a)^{2}(\sigma / a)^{2}$, vary by the largest amount, hence influencing the mean the most. The variations of these two terms are obviously greater than the first three. However, in the fourth term (as in the sixth), $m$ can range from 0 to $\infty$ and hence at first inspection the high values of $m$ would apparently cause the variations in the fourth term to dominate as well. However, by examining the series in Eq. (30), there is also severe attenuation due to radiation into the surrounding medium of this term in the limit of high $m$. For high $k a$, the term will be negligibly small before the effects on the fluctuations of the stochastic component of the term are realized. For example,

$$
2 \pi^{2} m^{2}(k a)^{2}(\sigma / a)^{2}\left[\beta_{R}^{\prime}(\infty)\right]^{2}=2(k a)^{2}(\sigma / a)^{2}
$$

when $m \simeq 137$. For this value of $m$ and $k a=10$, the attenuation term in Eq. (30) $e^{-2 \pi m k a \beta_{R}^{\prime}(\infty)} \simeq e^{-20} \simeq 2 \times 10^{-9}$. Note that this argument is also true for higher-order terms in the expansion. This can be demonstrated by comparing similar orders of terms 4 and 5 . The higher-order terms will only be comparable when $m$ is large, again beyond the point where suface elastic waves are important. Thus term 5 is shown to vary a larger amount than term 4 before radiation damping reduces the contributions due to term 4 . Also, since term 6 contains a factor of $m$ in it, it will dominate term 4 for all $m$ regardless of degree of radiation damping.

As shown above, terms 5 and 6 are the ones whose roughness-induced fluctuations are complex. They are the terms whose exponents are imaginary, hence the fluctuations are due to phase shifts in the local infinitesimal slices of the cylinder rather than amplitude variations. Since there is one of these complex terms in each of the two major terms in Eq. (30), then the mean of the entire expression is influenced the most by each of the complex terms, thus validating the approximation of ignoring variations due to the real terms in Eq. (31).

\section{Mean square}

In order to investigate the dominant terms of the mean square of the scattering amplitude, we square the separate terms and integrate them individually: 


$$
\begin{aligned}
\left.\left\langle\iint_{L / 2}^{L / 2}(\operatorname{term} 1) d z\right\}^{2}\right\rangle \\
=\int_{-L / 2}^{L / 2} \int_{-L / 2}^{L / 2}\left\langle(\text { term } 1)^{\prime}(\text { term } 1)^{\prime \prime}\right\rangle d z^{\prime} d z^{\prime \prime} \\
=k a \int_{-L / 2}^{L / 2} \int_{-L / 2}^{L / 2}\left[1-\frac{1}{4}\left(\frac{\sigma}{a}\right)^{2}+\frac{1}{4}\left(\frac{\sigma}{a}\right)^{2} \Phi(\xi)\right. \\
\left.\quad+\frac{1}{64}\left(\frac{\sigma}{a}\right)^{4}\left(1+2 \Phi^{2}(\xi)\right)\right] d z^{\prime} d z^{\prime \prime} \\
\simeq k a L^{2}\left[1-\frac{1}{4}\left(\frac{\sigma}{a}\right)^{2}\right]+\frac{1}{4} k a\left(\frac{\sigma}{a}\right)^{2} \\
\quad \times \int_{-L / 2}^{L / 2} \int_{-L / 2}^{L / 2} \Phi(\xi) d z^{\prime} d z^{\prime \prime} \\
=k a L^{2}\left\{1-\frac{1}{4}(\sigma / a)^{2}\right\} ;
\end{aligned}
$$

and similarly:

$$
\begin{aligned}
& \left\langle\left\{\int(\text { term 2) }\}^{2}\right)=(k a)^{2} L^{2},\right. \\
& \left\langle\iint(\text { term 3) }]^{2}\right) \\
& \quad \simeq L^{2}\left(1+4\left(\pi-\theta_{R}\right)^{2}(k a)^{2}\left[\beta_{R}^{\prime}(\infty)\right]^{2}(\sigma / a)^{2}\right)
\end{aligned}
$$

$$
\begin{aligned}
& \left\langle\left\{\int(\operatorname{term} 4)\right\}^{2}\right) \\
& \quad \simeq L^{2}\left(1+2 \pi^{2}\left(m^{\prime 2}+m^{\prime \prime 2}\right)(k a)^{2}\left[\beta_{R}^{\prime}(\infty)\right]^{2}(\sigma / a)^{2}\right),
\end{aligned}
$$

$\left\{\left\{\int(\right.\right.$ term 5$\left.\left.)\right\}\left\{\int(\text { term } 5)\right\}^{*}\right) \simeq L^{2}\left(1-4(k a)^{2}(\sigma / a)^{2}\right)$,

$$
\begin{aligned}
& \left\langle\left\{\int(\operatorname{term} 6)\right\}\left\{\int(\operatorname{term} 6)\right\}^{*}\right) \\
& \simeq L^{2}\left\{1-\frac{1}{2}(k a)^{2}(\sigma / a)^{2}\right. \\
& \left.\quad \times\left[\left(C_{R}+2 \pi m^{\prime} c / c_{R}\right)^{2}+\left(C_{R}+2 \pi m^{\prime \prime} c / c_{R}\right)^{2}\right]\right\},
\end{aligned}
$$

where the bivariate Gaussian defined in Eq. (A2) of Appen$\operatorname{dix} A$ was used in the double integral and the change of variables $\left(z^{\prime}, z^{\prime \prime}\right) \rightarrow(z, \xi)$ was made as performed in Eq. (B2) of Appendix B. Also, the integrals in Eqs. (A3)-(A8) and (B8) were used in the evaluation and terms involving $(\sigma / a)^{4}$ or powers of the ratio $\mathscr{L} / L(\ll 1)$ were found to be negligible.

For the same arguments as given with the means above, for $k \sigma, \sigma / a, \mathscr{L} / L \ll 1$, and $\beta_{R}^{\prime}(\infty) \ll 1$, fractional variations in the mean square of the scattering amplitude are dominated by the complex terms 5 and 6 . Thus, again, it is the phase shifts of the infinitesimal slices of the cylinder in the integrand that dominate the averaged field, where this time the average is of the square. The analysis to this point in this section explicitly justifies ignoring the amplitude variation terms in Eq. (30) for calculating the mean square in Eq. (43) for this low roughness case, $k \sigma, \sigma / a \ll 1$.

While the analysis in this Appendix explicitly involves a low roughness limit of the various terms of $\mathrm{Eq}$. (30), the mean and mean square results can be generalized to the finite roughness case of $k \sigma \gtrsim 1$, although $\sigma / a$ should remain much less than unity so the object does not break. For this finite roughness case, many more terms are involved in the expansions in Eqs. (C1) and (C3)-(C6). In each case, there will be higher-order powers of the expansion terms shown, and as in the $k \sigma \ll 1$ case, the expansion terms of the complex terms "term 5" and "term 6" are much greater than the other terms hence resulting in the complex terms dominating the variations of the mean and mean square of $\mathrm{Eq}$. (30). Finally, while the analysis in this Appendix explicitly involves the individual terms of Eq. (30), a similar analysis (not shown) investigating the dominant terms of the products and resultant cross product terms produce the same results-variations in the complex terms dominate the means of Eq. (30).

In conclusion, regardless of the region, $k \sigma \ll 1$ or $k \sigma \gtrsim 1$, the complex (phase shift) terms 5 and 6 dominate roughness-induced variations in the mean and mean-square scattered field.
' $V$. Twersky, "On the non-specular reflection of plane waves of sound," $J$. Acoust. Soc. Am. 22, 539-546 (1950).

${ }^{2} \mathrm{~V}$. Twersky, "On scattering and reflection of sound by rough surfaces," $\mathrm{J}$. Acoust. Soc. Am. 29, 209-225 (1957).

${ }^{3}$ C. F. Greenlaw, "Backscattering spectra of preserved zooplankton," $\mathbf{J}$. Acoust. Soc. Am., 62, 44-52 (1977).

${ }^{4}$ R. E. Pieper and D. V. Holliday, "Acoustic measurements of zooplankton distribution in the sea," J. Cons. Int. Explor. Mer. 41, 226-238 (1984).

${ }^{5} \mathrm{~K}$. E. Richter, " $1.2 \mathrm{MHz}$ acoustic scattering from individual zooplankters and copepod populations," Deep-Sea Res. 32(2A), 149-161 (1985).

"A. Kristensen and J. Dalen, "Acoustic estimation of size distribution and abundance of zooplankton," J. Acoust. Soc. Am. 80, 601-61I (1986).

'J. Dalen and K. Kristensen, "Comparative studies of theoretical and empirical target strength models of euphausiids (krill) in relation to field experiment data," International Symposium on Fisheries Acoustics, Seattle, Washington, 22-26 June 1987.

J. Sheng and A. E. Hay, "An examination of the spherical scatterer approximation in aqueous suspensions of sand," J. Acoust. Soc. Am. 83, $598-610$ (1988).

${ }^{9} \mathrm{~T}$. K. Stanton, "Sound scattering by cylinders of finite length. I. Fluid cylinders," J. Acoust. Soc. Am. 83, 55-63 (1988).

${ }^{10}$ T. K. Stanton, "Sound scattering by cylinders of finite length. I1I. Deformed cylinders," J. Acoust. Snc. Am. 86, 691-705 (1989).

${ }^{11}$ T. K. Stanton, "Simple approximate formulas for backscattering of sound by spherical and elongated objects," J. Acoust. Soc. Am. 86, 1499-1510 (1989).

${ }^{12}$ T. K. Stanton, "Sound scattering by zooplankton," Rapp. P.-V. Reun. Cons. Int. Explor. Mer. 189, 353-362 (1990).

${ }^{13}$ C. S. Clay, "Low-resolution acoustic scattering models: Fluid-filled cylinders and fish wilh swimbladders," J. Acoust. Soc. Am. 89, 2168-2179 (1991).

${ }^{14}$ P. H. Wiebe, C. H. Greene, T. K. Stanton, and J. Burczynski, "Sound scattering by live zooplankton and micronekton: Empirical studies with a dual-beam acoustical system," J. Acoust. Soc. Am. 88, 2346-2360 (1990).

${ }^{15}$ D. Chu, T. K. Stanton, and P. H. Wiebe, "Frequency dependence of sound backscattering from live individual zooplankton," ICES J. Mar. Sci. 49, 97-106 ( 1992).

${ }^{16}$ P. M. Morse and H. Feshback, Methads of Theoretical Physics (Part II) (McGraw-Hill, New York, 1953), Chap. 9.2.

I7. B. Mehl, "Acoustic resonance frequencies of deformed spherical resonators. II," J. Acoust. Soc. Am. 79, 278-285 (1986).

${ }^{18}$ F. G. Bass and I. M. Fuks, Wave Scattering from Statistically Rough Surfaces, edited and translated by C. B. Vesecky and J. F. Vesecky (Pergamon, Oxford, 1979). 
${ }^{19}$ Yu. Yu. Popov, "Sound scattering by a statistically corrugated cylindrical surface," Sov. Phys. Acoust. 31(6), 509-511 (1985).

${ }^{20} \mathrm{Yu}$. Yu. Popov, "Propagation of creeping waves around a statistically rough cylindrical surface," Sov. Phys. Acoust. 33(2), 189-192 (1987).

${ }^{21}$ Y. M. Chen, "Diffraction by a smooth transparent object," J. Math Phys. 5(6), 820-832 (1964).

${ }^{22} \mathrm{P}$. Barber and C. Yeh, "Scattering of electromagnetic waves by arbitrarily shaped dielectric bodies," Appl. Opt. 14, 2864-2872 (1975).

${ }^{23}$ V. K. Varadan, V. V. Varadan, and Y.-H. Pao, "Multiple scattering of elastic waves by cylinders of arbitrary cross section. I. SH waves," J. Acoust. Soc. Am. 63, 1310-1319 (1978).

${ }^{24} \mathrm{H}$. M. Nussenzveig, "High frequency scattering by an impenetrable sphere," Ann. Phys. 34, 23-95 (1965).

${ }^{25}$ H. M. Nussenzveig, "High frequency scattering by a transparent sphere. I. Direct reflection and transmission," J. Math. Phys. 10, 82-124 (1969).

${ }^{26}$ H. M. Nussenzveig, "High frequency scattering by a transparent sphere. II. Theory of the rainbow and glory," J. Math. Phys. 10, 125-176 (1969).

${ }^{27}$ H. Überall, R. D. Doolittle, and J. V. McNicholas, "Use of sound pulses for a study of circumferential waves," J. Acoust. Soc. Am. 39, 564-578 (1966).

${ }^{28}$ R. D. Doolittle, H. Überall, and P. Uginčius, "Sound scattering by elastic cylinders," J. Acoust. Soc. Am. 43, 1-14 (1968).

${ }^{29} \mathrm{P}$. Uginčius and $\mathrm{H}$. Überall, "Creeping-wave analysis of acoustic scattering by elastic cylindrical shells," J. Acoust. Soc. Am. 43, 1025-1035 (1968).

${ }^{30}$ R. E. Bunney, R. R. Goodman, and S. W. Marshall, "Rayleigh and Lamb waves on cylinders," J. Acoust. Soc. Am. 46, 1223-1233 (1969).

${ }^{31}$ D. Brill and H. Überall, "Acoustic waves transmitted through elastic cylinders," J. Acoust. Soc. Am. 50, $921-939$ (1971).

${ }^{32}$ H. Überall, "Surface waves in acoustics," in Physical Acoustics, edited by W. P. Mason and R. N. Thurston (Academic, New York, 1973), Vol. 10, pp. 1-60.

${ }^{33}$ G. V. Frisk, J. W. Dickey, and H. Überall, "Surface wave modes on elastic cylinders," J. Acoust. Soc. Am. 58, 996-1008 (1975).

${ }^{34}$ J. W. Dickey, G. V. Frisk, and H. Überall, "Whispering Gallery wave modes on elastic cylinders," J. Acoust. Soc. Am. 59, 1339-1346 (1976).

${ }^{35} \mathrm{G}$. V. Frisk and H. Überall, "Creeping waves and lateral waves in acoustic scattering by large cylinders," J. Acoust. Soc. Am. 59, 46-54 (1976).

${ }^{36} \mathrm{~J}$. W. Dickey and H. Überall, "Acoustic high-frequency scattering by elastic cylinders," J. Acoust. Soc. Am. 66, 275-283 (1979).

${ }^{31}$ K. L. Williams and P. L. Marston, "Backscattering from an elastic sphere: Sommerfeld-Watson transformation and experimental confirmation," J. Acoust. Soc. Am. 78, 1093-1 102 (1985).

${ }^{38}$ K. L. Williams and P. L. Marston, "Synthesis of backscattering from an elastic sphere using the Sommerfeld-Watson transformation and giving a Fabry-Perot analysis of resonances," J. Acoust. Soc. Am. 79, 1702-1708 (1986).

${ }^{39}$ K. L. Williams and P. L. Marston, "Axially focused (glory) scattering due to surface waves generated on spheres: Model and experimental confirmation using tungsten carbide spheres," J. Acous. Soc. Am. 78, 722728 (1985).

${ }^{40}$ P. L. Marston, "GTD for backscattering from elastic spheres and cylinders in water and the coupling of surface elastic waves with the acoustic field," J. Acoust. Soc. Am. 83, 25-37 (1988).
${ }^{41}$ E. Heyman and L. Felsen, "Creeping waves and resonances in transient scattering by smooth convex objects”, IEEE Trans. Antenna Propag. AP-31, 426-437 (1983).

${ }^{42}$ L. B. Felsen, J. M. Ho, and I. T. Lu, "Three-dimensional Green's function for fluid-loaded thin elastic cylindrical shell: Formulation and solution," J. Acoust. Soc. Am. 87, 543-553 (1990).

${ }^{43}$ L. B. Felsen, J. M. Ho, and I. T. Lu, "Three-dimensional Green's function for fluid-loaded thin elastic cylindrical shell: Alternative representations and ray acoustic forms," J. Acoust. Soc. Am. 87, 554-569 (1990).

${ }^{4}$ N. D. Veksler and V. M. Korsunskii, "On the acoustic pressure formula for backscattering by thin elastic circular cylindrical shells," Acoust. Lett. 6, 70-78 (1982).

${ }^{45}$ G. C. Gaunaurd and M. F. Werby, "Lamb and creeping waves around submerged spherical shells resonantly excited by sound scattering," $J$. Acoust. Soc. Am. 82, 2021-2033 (1987).

${ }^{46} \mathrm{C}$. Eckart, "The scattering of sound from the sea surface," $J$. Acoust. Soc. Am. 25, 566-570 (1953).

${ }^{47}$ J. M. Proud, Jr., R. T. Beyer, and P. Tamarkin, "Reflection of sound from randomly rough surfaces," J. Appl. Phys. 31, 543-552 (1960).

${ }^{48}$ D. R. Melton and C. W. Horton, Sr., "Importance of the Fresnel Correction in scattering from a rough surface. I. Phase and amplitude fluctuations," J. Acoust. Soc. Am. 47, 290-298 (1970).

${ }^{49} \mathrm{C}$. S. Clay and H. Medwin, "Dependence of spatial and temporal correlation of forward scattered underwater sound on the surface statistics." I. Theory. J. Acoust. Soc. Am. 47, 1412-1418 (1970); and II. Experiment. J. Acoust. Soc. Am. 47, 1419-1429 (1970).

${ }^{\text {so }}$ C. S. Clay and H. Medwin, Acoustical Oceanography: Principles and Applications (Wiley-Interscience, New York, 1977).

si C. S. Clay and W. K. Leong, "Acoustic estimates of topography and roughness spectrum of the sea floor southwest of Iberian Peninsula," in Physics of Sound in Marine Sediments, edited by L. Hampton (Plenum, New York, 1974) (summarized in Ref. 50).

${ }^{52}$ P. D. Thorne and N. G. Pace, "Acoustic studies of broadband scattering from a model rough surface," J. Acoust. Soc. Am. 75, 133-144 (1984).

${ }^{53}$ T. K. Stanton, "Sonar estimates of sea floor microroughness," J. Acoust. Soc. Am. 75, 809-818 (1984)

${ }^{54}$ T. K. Stanton, K. C. Jezek, and A. J. Gow, "Acoustical reflection and scattering from the underside of laboratory grown sea ice: Measurements and predictions," J. Acoust. Soc. Am. 80, 1486-1494 (1986).

${ }^{55}$ T. K. Stanton, "Sound scattering by rough elongated elastic objects. II: Fluctuations of scattered field," J. Acoust. Soc. Am. 92, 1665-1678 (1992).

${ }^{56}$ E. Skudrzyk, The Foundations of Acoustics (Springer-Verlag, New York, 1971).

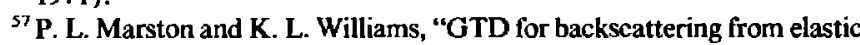
objects in water: Phase of the coupling coefficient and a simplified synthesis of the form function," J. Acoust. Soc. Am. Suppl. 1 83, S94 (1988).

${ }^{58}$ G. F. Carrier, M. Krook, and C. E. Pearson, Functions of a Complex Variable (McGraw-Hill, New York, 1966).

${ }^{59}$ G. W. C. Kaye and T. H. Laby, Tables of Physical and Chemical Constants (Longman, New York, 1973).

${ }^{60}$ S. O. Rice, "Mathematical analysis of random noise," in Selected Papers on Noise and Stochastic Processes, edited by N. Wax (Dover, New York, 1954), pp. 133-294. 